\title{
Actions and identities on set partitions
}

\author{
Eric Marberg \\ Department of Mathematics \\ Massachusetts Institute of Technology \\ emarberg@math.mit.edu
}

Submitted: Sep 23, 2011; Accepted: Jan 11, 2012; Published: Jan 27, 2012

Mathematics Subject Classification: 05A18, 05E18

\begin{abstract}
A labeled set partition is a partition of a set of integers whose arcs are labeled by nonzero elements of an abelian group $\mathbb{A}$. Inspired by the action of the linear characters of the unitriangular group on its supercharacters, we define a group action of $\mathbb{A}^{n}$ on the set of $\mathbb{A}$-labeled partitions of an $(n+1)$-set. By investigating the orbit decomposition of various families of set partitions under this action, we derive new combinatorial proofs of Coker's identity for the Narayana polynomial and its type B analogue, and establish a number of other related identities. In return, we also prove some enumerative results concerning André and Neto's supercharacter theories of type B and D.
\end{abstract}

\section{Introduction}

A set partition is formally a set of nonempty, pairwise disjoint sets, which we always assume consist of integers and which we refer to as blocks. We call a pair of integers $(i, j)$ an arc of a set partition if $i$ and $j$ occur in the same block and $j$ is the least element of the block greater than $i$. Let $\operatorname{Arc}(\Lambda)$ denote the set of arcs of a set partition $\Lambda$.

We write $\Lambda \vdash \mathcal{X}$ and say that $\Lambda$ is a partition of a set $\mathcal{X}$ if $\Lambda$ is a set partition the union of whose blocks is $\mathcal{X}$. The standard representation of a partition $\Lambda \vdash \mathcal{X}$ is then the directed graph with vertex set $\mathcal{X}$ and edge set $\operatorname{Arc}(\Lambda)$, drawn by listing the elements of $\mathcal{X}$ in order with the corresponding arcs overhead. For example, the set partitions $\Lambda=\{\{1,3,4,7\},\{2,6\},\{5\}\}$ and $\Gamma=\{\{1,7\},\{2,3,4,6\},\{5\}\}$ have standard representations

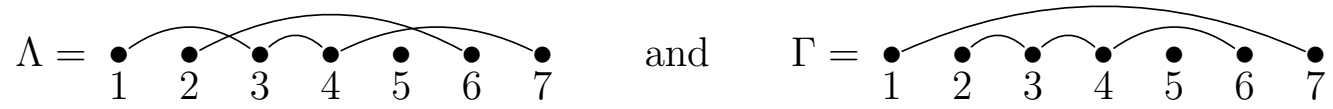

since $\operatorname{Arc}(\Lambda)=\{(1,3),(2,6),(3,4),(4,7)\}$ and $\operatorname{Arc}(\Gamma)=\{(1,7),(2,3),(3,4),(4,6)\}$. A set partition $\Lambda$ is noncrossing if no two $\operatorname{arcs}(i, k),(j, l) \in \operatorname{Arc}(\Lambda)$ have $i<j<k<l$, which means that no arcs cross in its standard representation. 
This paper investigates a group action on set partitions which are labeled in the following sense. Given an additive abelian group $\mathbb{A}$, an $\mathbb{A}$-labeled set partition is a set partition $\Lambda$ with a map $\operatorname{Arc}(\Lambda) \rightarrow \mathbb{A} \backslash\{0\}$, denoted $(i, j) \mapsto \Lambda_{i j}$. This is essentially the definition of a colored rhyming scheme as studied in [31], except that we require the colors to form the set of nonzero elements of an abelian group. For each nonnegative integer $n$, we define

$$
\begin{aligned}
\Pi(n, \mathbb{A}) & :=\text { the set of } \mathbb{A} \text {-labeled partitions of }[n]:=\{i \in \mathbb{Z}: 1 \leq i \leq n\} \\
\mathrm{NC}(n, \mathbb{A}) & :=\text { the set of } \mathbb{A} \text {-labeled noncrossing partitions of }[n], \\
\mathrm{L}(n, \mathbb{A}) & :=\text { the set of } \mathbb{A} \text {-labeled partitions of }[n] \text { with consecutive integer blocks. }
\end{aligned}
$$

Note that an $\mathbb{A}$-labeled partition $\Lambda \vdash[n]$ belongs to $\mathrm{L}(n, \mathbb{A})$ if and only if every arc of $\Lambda$ has the form $(i, i+1)$ for some $i \in[n-1]$. We begin by defining an operation of $\mathrm{L}(n, \mathbb{A})$ on $\Pi(n, \mathbb{A})$.

Definition 1.1. Given $\alpha \in \mathrm{L}(n, \mathbb{A})$ and $\Lambda \in \Pi(n, \mathbb{A})$, define $\alpha+\Lambda$ as the $\mathbb{A}$-labeled partition of $[n]$ whose standard representation is obtained by the following procedure:

- List the numbers $1,2, \ldots, n$ and draw the labeled arcs of both $\alpha$ and $\Lambda$ overhead.

- Whenever two arcs coincide, add their labels and replace the pair with a single arc.

- Whenever two distinct arcs share an endpoint, delete the shorter arc.

- Finally, remove any arcs labeled by zero.

For example,

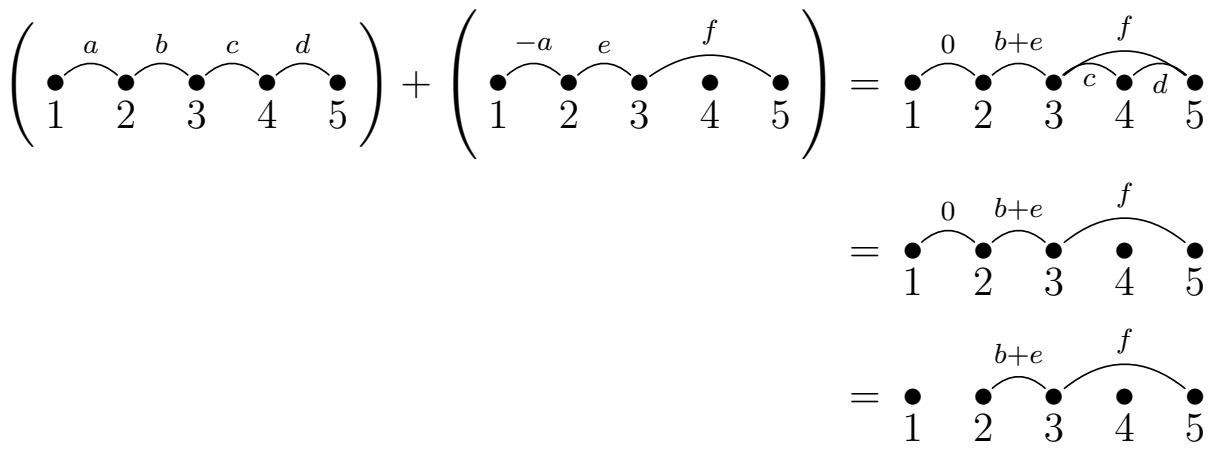

for $a, b, c, d, e, f \in \mathbb{A} \backslash\{0\}$ with $b \neq-e$.

The operation + gives $\mathrm{L}(n, \mathbb{A})$ the structure of an abelian group isomorphic to $\mathbb{A}^{n-1}$ which acts on both $\Pi(n, \mathbb{A})$ and $\mathrm{NC}(n, \mathbb{A})$. This action has several interesting properties and serves as a useful tool for providing succinct combinatorial proofs of some notable identities. The following is a motivating example. In studying some enumerative problems associated with a class of lattice paths, Coker [17] derived, using generating functions and 
the Lagrange inversion formula, the equivalence of two expressions for the rank generating function of the lattice of noncrossing partitions of type $A_{n}$. This amounted to the identity

$$
\sum_{k=0}^{n} \frac{1}{n+1}\left(\begin{array}{c}
n+1 \\
k
\end{array}\right)\left(\begin{array}{c}
n+1 \\
k+1
\end{array}\right) x^{k}=\sum_{k=0}^{\lfloor n / 2\rfloor} \mathcal{C}_{k}\left(\begin{array}{c}
n \\
2 k
\end{array}\right) x^{k}(1+x)^{n-2 k},
$$

with $\mathcal{C}_{k}=\frac{1}{k+1}\left(\begin{array}{c}2 k \\ k\end{array}\right)$ denoting the $k$ th Catalan number. Somewhat earlier, Riordan included in his book [30] a similar equation involving the rank generating function of the lattice of noncrossing partitions of type $B_{n}$ :

$$
\sum_{k=0}^{n}\left(\begin{array}{l}
n \\
k
\end{array}\right)^{2} x^{k}=\sum_{k=0}^{\lfloor n / 2\rfloor}\left(\begin{array}{c}
2 k \\
k
\end{array}\right)\left(\begin{array}{c}
n \\
2 k
\end{array}\right) x^{k}(x+1)^{n-2 k} .
$$

We obtain a simple combinatorial proof of 1.2 from Definition 1.1 by noting that when $x=|\mathbb{A}|-1$, the terms in the left sum count the elements of $\mathrm{NC}(n+1, \mathbb{A})$ with $n-k$ blocks, while the terms in the right sum count the elements of $\mathrm{NC}(n+1, \mathbb{A})$ whose $\mathrm{L}(n+1, \mathbb{A})$ orbits have size $|\mathbb{A}|^{n-2 k}$. The second identity $(1.3)$ follows by the same argument applied to a certain family of "type B" A-labeled set partitions; see the remarks to Theorems 3.4 and 6.7 below.

Remark. Two recent papers have supplied combinatorial proofs for $(1.2)$ and $(1.3)$ using quite different methods. In [16], Chen, Yan, and Yang prove (1.2) by inspecting a weighted version of a bijection between Dyck paths and 2-Motzkin paths; in [15], Chen, Wang, and Zhang prove $(1.3)$ by enumerating certain weighted type B noncrossing partitions. Algebraic proofs of these identities are much easier to come by: as pointed out by Christian Krattenthaler, (1.2) and (1.3) are respectively the special cases $(a, b)=(-n,-n-1)$ and $(a, b)=(-n,-n)$ of the quadratic transformation formula

$$
{ }_{2} F_{1}(a, b ; 1+a-b ; x)=\frac{{ }_{2} F_{1}\left(\frac{a}{2}, \frac{a}{2}+\frac{1}{2} ; 1+a-b ; \frac{4 x}{(1+x)^{2}}\right)}{(1+x)^{a}}
$$

for the hypergeometric function [21, Eq. 2.11(34)]. This more general identity has been known since at least 1881, when it appeared in an equivalent form as [22, Eq. (36)].

Definition 1.1 is motivated by the representation theory of $\mathbf{U}_{n}\left(\mathbb{F}_{q}\right)$, the group of $n \times n$ unipotent upper triangular matrices over a finite field with $q$ elements. In detail, the $\mathbb{F}_{q}$-labeled partitions of $[n]$ naturally index the supercharacters of $\mathbf{U}_{n}\left(\mathbb{F}_{q}\right)$, a certain family of complex characters whose constituents partition the set of the group's irreducible characters and which have a number of other notable properties (see [39] for a concise overview). Given $\lambda \in \Pi\left(n, \mathbb{F}_{q}\right)$, let $\chi_{\lambda}$ denote the associated supercharacter (see Section 7 below for an explicit definition). The correspondence $\lambda \mapsto \chi_{\lambda}$ then defines a bijection from $\mathrm{L}\left(n, \mathbb{F}_{q}\right)$ to the set of linear characters of $\mathbf{U}_{n}\left(\mathbb{F}_{q}\right)$, and if $\alpha \in \mathrm{L}\left(n, \mathbb{F}_{q}\right)$ and $\lambda \in \Pi\left(n, \mathbb{F}_{q}\right)$ then the product of the characters $\chi_{\alpha}$ and $\chi_{\lambda}$ is precisely $\chi_{\alpha+\lambda}$ (see [39, Corollary 4.7]). If general, if $\lambda, \mu \in \Pi\left(n, \mathbb{F}_{q}\right)$ then the product of $\chi_{\lambda}$ and $\chi_{\mu}$ is a linear 
combination $\sum_{\nu \in \Pi\left(n, \mathbb{F}_{q}\right)} c_{\lambda \mu}^{\nu} \chi_{\nu}$ for some nonnegative integers $c_{\lambda \mu}^{\nu}$. Finding a combinatorial rule to determine these coefficients is an open problem, notably studied in [25].

We organize this article as follows. In Section 2 we reexamine Definition 1.1 in slightly greater detail and introduce a few useful conventions. We carry out a careful analysis of the labeled set partition orbits under our action in Section 3, and use this to give combinatorial proofs of several identities in the style of $(1.2)$ and $(1.3)$. Sections 4 and 5 introduce type B and D analogues for the family of labeled set partitions studied in Section 3, and in Section 6 we undertake a similar orbit analysis to prove analogues of our classical identities in these other types. In Section 7, we explore the connection between our methods and the supercharacters of the unitriangular group more closely. In particular, we provide explicit, succinct definitions of André and Neto's supercharacters of type B and D, and compute the sizes of several natural families of these characters.

\section{Two equivalent definitions}

In this preliminary section we note two equivalent characterizations of the operation + presented in Definition 1.1, and show how this operation leads to another proof of the rank symmetry of the lattice of noncrossing partitions. We begin with the following observation, whose derivation from Definition 1.1 is a straightforward exercise.

Observation 2.1. Given $\alpha \in \mathrm{L}(n, \mathbb{A})$ and $\Lambda \in \Pi(n, \mathbb{A})$, let

$$
\begin{aligned}
& \mathcal{S}=\left\{(j, j+1) \in \operatorname{Arc}(\alpha) \cap \operatorname{Arc}(\Lambda): \alpha_{j, j+1}+\Lambda_{j, j+1}=0\right\} \\
& \mathcal{T}=\{(j, j+1) \in \operatorname{Arc}(\alpha):(i, j+1) \notin \operatorname{Arc}(\Lambda) \text { and }(j, \ell) \notin \operatorname{Arc}(\Lambda) \text { for all } i, \ell\} .
\end{aligned}
$$

Then $\alpha+\Lambda$ is the element of $\Pi(n, \mathbb{A})$ with $\operatorname{arc}$ set $(\operatorname{Arc}(\Lambda)-\mathcal{S}) \cup \mathcal{T}$ and labeling map

$$
(\alpha+\Lambda)_{j k}= \begin{cases}\alpha_{j k}+\Lambda_{j k}, & \text { if }(j, k) \in \operatorname{Arc}(\alpha) \cap \operatorname{Arc}(\Lambda)-\mathcal{S} \\ \alpha_{j k}, & \text { if }(j, k) \in \mathcal{T} \\ \Lambda_{j k}, & \text { otherwise }\end{cases}
$$

This observation makes clear that $\alpha+\Lambda$ differs from $\Lambda$ only in its arcs of the form $(j, j+1)$. Such arcs are never involved in crossings, and so the action of $\mathrm{L}(n, \mathbb{A})$ on $\Pi(n, \mathbb{A})$ preserves $\mathrm{NC}(n, \mathbb{A})$; i.e., $\alpha+\Lambda \in \mathrm{NC}(n, \mathbb{A})$ for all $\alpha \in \mathrm{L}(n, \mathbb{A})$ and $\Lambda \in \mathrm{NC}(n, \mathbb{A})$.

There is a useful bijection from $\Pi(n, \mathbb{A})$ to the set of $n \times n$ matrices over $\mathbb{A}$ which are strictly upper triangular and have at most one nonzero entry in each row and column. The matrix or rook diagram of $\Lambda \in \Pi(n, \mathbb{A})$ is the $n \times n$ matrix with the entry $\Lambda_{i j}$ in position $(i, j)$ for each $(i, j) \in \operatorname{Arc}(\Lambda)$ and zeros elsewhere. A set partition is noncrossing if and only if there is no position above the diagonal in its associated matrix which is both strictly south of a nonzero entry in the same column and strictly west of a nonzero entry in the same row. Likewise, a set partition belongs to $\mathrm{L}(n, \mathbb{A})$ if and only if its matrix has nonzero positions only on the superdiagonal $\{(i, i+1): i \in[n-1]\}$. This fact shows that we may also equivalently define the operation + as follows: 
Observation 2.2. If $\alpha \in \mathrm{L}(n, \mathbb{A})$ and $\Lambda \in \Pi(n, \mathbb{A})$ then $\alpha+\Lambda$ is the element of $\Pi(n, \mathbb{A})$ produced by the following procedure:

1. Add the matrices of $\alpha$ and $\Lambda$ to form a matrix $M$ over $\mathbb{A}$.

2. Replace with zero any nonzero positions $(i, i+1)$ on the superdiagonal of $M$ which lie strictly below a nonzero position in the same column or strictly to the left of a nonzero position in the same row.

3. Define $\alpha+\Lambda$ to be the element of $\Pi(n, \mathbb{A})$ associated to the modified matrix $M$.

This formulation of Definition 1.1 most clearly illustrates that the addition + makes $\mathrm{L}(n, \mathbb{A})$ into an abelian group acting on $\Pi(n, \mathbb{A})$ : the group is just the additive group of $n \times n$ matrices over $\mathbb{A}$ whose nonzero entries are all on the superdiagonal.

Let $\Pi(n)$ and $\mathrm{NC}(n)$ denote the sets of ordinary and noncrossing (unlabeled) partitions of $[n]$. We may view the elements of $\Pi(n)$ and $\operatorname{NC}(n)$ as $\mathbb{A}$-labeled set partitions by taking $\mathbb{A}=\mathbb{F}_{2}$ to be a finite field with two elements. These sets are partially ordered by refinement: $\Gamma \leq \Lambda$ if each block of $\Gamma \vdash[n]$ is contained in some block of $\Lambda \vdash[n]$. This partial order makes $\Pi(n)$ and $\mathrm{NC}(n)$ into graded lattices with height $n-1$ according to the rank function $\operatorname{rank}(\Lambda)=n-|\Lambda|$. The lattice $\operatorname{NC}(n)$ in particular has a number of remarkable properties and an extensive literature (see [5] for a survey).

As a first application of the action of $\mathrm{L}(n, \mathbb{A})$ on $\Pi(n, \mathbb{A})$, we note that the map

$$
\Lambda \mapsto\{\{1,2, \ldots, n\}\}+\Lambda
$$

defines an involution of the set of $\left(\mathbb{F}_{2}\right.$-labeled) partitions of $[n]$. We denote the image of $\Lambda \vdash[n]$ under this involution by $\Lambda^{+}$; the latter partition has the following explicit definition, which makes sense even for partitions of sets other than $[n]$.

Definition 2.3. Given a set $\mathcal{X} \subset \mathbb{Z}$ and a partition $\Lambda \vdash \mathcal{X}$, let $\Lambda^{+}$be the partition of $\mathcal{X}$ with arc set $(\operatorname{Arc}(\Lambda)-\mathcal{S}) \cup \mathcal{T}$, where $\mathcal{S}=\{(i, i+1): i \in \mathbb{Z}\}$ and $\mathcal{T}$ is the set of pairs $(i, i+1)$ in $\mathcal{X} \times \mathcal{X}$ with the property that $i$ and $i+1$ are respectively maximal and minimal in their blocks of $\Lambda$.

For example, we have $\{\{1,4,5\},\{2,3\},\{6,7\},\{8\}\}^{+}=\{\{14\},\{2\},\{3\},\{5,6\},\{7,8\}\}$. Our main point in presenting this involution is simply to note that it gives another proof of the fact that the lattice $\mathrm{NC}(n)$ is rank symmetric.

Proposition 2.4. The map $\Lambda \mapsto \Lambda^{+}$is rank inverting on $\mathrm{NC}(n)$. That is, if $\Lambda \in \mathrm{NC}(n)$ has $k$ blocks, then $\Lambda^{+} \in \mathrm{NC}(n)$ has $n+1-k$ blocks.

The involution is not order reversing on $\mathrm{NC}(n)$, since for example

$$
\{\{1\},\{2,3\},\{4\}\}^{+}=\{\{1,2\},\{3,4\}\} \ngtr\{\{1,4\},\{2\},\{3\}\}=\{\{1,4\},\{2,3\}\}^{+} .
$$

Also, the property $\left|\Lambda^{+}\right|=n+1-|\Lambda|$ may fail if $\Lambda \vdash[n]$ is not noncrossing. 
Proof. Fix $\Lambda \in \mathrm{NC}(n)$ with $k$ blocks. If $\{n\}$ is a block of $\Lambda$ and $\Lambda^{\prime} \in \mathrm{NC}(n-1)$ is the set partition formed by removing this block, then $\Lambda^{+}$is given by adding $n$ to the block of $n-1$ in $\left(\Lambda^{\prime}\right)^{+}$. Hence the number of blocks of $\Lambda^{+}$is the same as the number of blocks of $\left(\Lambda^{\prime}\right)^{+}$, which by induction is $(n-1)+1-(k-1)=n+1-k$ as desired.

Suppose the block of $n$ in $\Lambda$ has more than one element. In this block, $n$ is the largest element; let $m$ be the second largest so that $(m, n) \in \operatorname{Arc}(\Lambda)$. Let $A$ be the noncrossing partition of $[m]$ with $\operatorname{arc} \operatorname{set} \operatorname{Arc}(\Lambda) \cap\{(i, j): 1 \leq i<j \leq m\}$ and let $B$ be the noncrossing partition of $[n-1] \backslash[m]$ with arc set $\operatorname{Arc}(\Lambda) \cap\{(i, j): m<i<j<n\}$. Observe that $A \cup B$ is then a partition of $[n-1]$, and that because $\Lambda$ is noncrossing, adding $n$ to the block of $m$ in $A \cup B$ recovers $\Lambda$.

If $m=n-1$ then $|A|=k$ and $B=\varnothing$ and $\Lambda^{+}=A^{+} \cup\{\{n\}\}$, so the number of blocks of $\Lambda^{+}$is $1+\left|A^{+}\right|=1+(n-1)+1-|A|=n+1-k$ by induction. Alternatively, if $m<n-1$ then $\Lambda^{+}$is formed by adding $n$ to the block of $m$ in $A^{+} \cup B^{+}$. Thus the number of blocks of $\Lambda^{+}$is $\left|A^{+} \cup B^{+}\right|=m+1-|A|+(n-1-m)+1-|B|=n+1-|A|-|B|=n+1-k$, again by induction.

\section{Identities for classical set partitions}

In this section we examine the action of $\mathrm{L}(n, \mathbb{A})$ on $\Pi(n, \mathbb{A})$ and $\mathrm{NC}(n, \mathbb{A})$ in greater detail. To begin, we note that shifting the matrix of a set partition one column to the right corresponds to an injective map

$$
\text { shift }: \Pi(n, \mathbb{A}) \rightarrow \Pi(n+1, \mathbb{A})
$$

which assigns $\Lambda \in \Pi(n, \mathbb{A})$ to the $\mathbb{A}$-labeled set partition of $[n+1]$ with arc set $\{(i, j+1)$ : $(i, j) \in \operatorname{Arc}(\Lambda)\}$ and labeling map $(i, j+1) \mapsto \Lambda_{i j}$. For example,

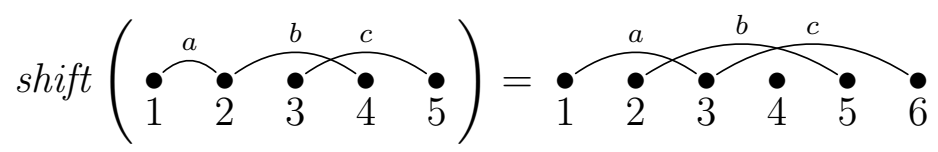

for $a, b, c \in \mathbb{A} \backslash\{0\}$. The map shift increases the number of blocks of a set partition by one, and its image consists of all $\mathbb{A}$-labeled partitions of $[n+1]$ with no blocks containing both $i$ and $i+1$ for some $i \in[n]$; following [13], we call such set partitions 2-regular. The right inverse of shift (defined on the matrix of a set partition by deleting the first column and last row then setting all diagonal entries to zero) is precisely the "reduction algorithm" presented in [13]; see also [23].

We say that a set partition of $[n]$ is feasible if each of its blocks has at least two elements and poor if each of its blocks has at most two elements. The matrix of a feasible set partition has a nonzero entry in either the $i$ th row or $i$ th column for each $i \in[n]$, while the matrix of a poor set partition never has a nonzero entry in both the $i$ th row and $i$ th column. From these considerations, it is straightforward to deduce the following lemma.

Lemma 3.1. The following restrictions of shift are bijections: 
(1) $\{$ Feasible partitions of $[n]\} \rightarrow\left\{\begin{array}{l}\text { 2-regular partitions } \Lambda \vdash[n+1] \text { such that } \\ 1+\max B \neq \min B^{\prime} \text { for all blocks } B, B^{\prime} \in \Lambda\end{array}\right\}$.

(2) $\{$ Poor noncrossing partitions of $[n]\} \rightarrow\{$ 2-regular noncrossing partitions of $[n+1]\}$.

To count feasible and poor set partitions, we introduce the polynomials

$$
\mathcal{F}_{n}(x):=\sum_{\text {Feasible } \Lambda \in \Pi(n)} x^{|\operatorname{Arc}(\Lambda)|} \quad \text { and } \quad \mathcal{M}_{n}(x):=\sum_{\operatorname{Poor} \Lambda \in \operatorname{NC}(n)} x^{|\operatorname{Arc}(\Lambda)|} .
$$

We can write $\mathcal{F}_{n}(x)=\sum_{k=0}^{n}\left\{\left\{\begin{array}{l}n \\ k\end{array}\right\}\right\} x^{n-k}$ where $\left\{\left\{\begin{array}{l}n \\ k\end{array}\right\}\right\}$ is the associated Stirling number of the second kind [19], which counts the number of feasible set partitions of $[n]$ with $k$ blocks and is listed as sequence [33, A008299]. The numbers $\mathcal{F}_{n}(1)$ themselves give sequence [33, A000296].

Letting $\mathcal{C}_{n}:=\frac{1}{n+1}\left(\begin{array}{c}2 n \\ n\end{array}\right)$ as in the introduction, we have the following slightly more explicit expression for $\mathcal{M}_{n}(x)$. The functions $\mathcal{M}_{n}(x)$ are sometimes called Motzkin polynomials (see [33, A055151]) and $\mathcal{M}_{n}(1)$ is the $n$th Motzkin number [33, A001006].

Proposition 3.2. $\mathcal{M}_{n}(x)=\sum_{k=0}^{\lfloor n / 2\rfloor} \mathcal{C}_{k}\left(\begin{array}{c}n \\ 2 k\end{array}\right) x^{k}$ for $n \geq 0$.

Proof. Poor noncrossing set partitions of $[n]$ with $k$ arcs are in bijection with pairs $(S, \Lambda)$, where $S$ is a $2 k$-subset of $[n]$ and $\Lambda$ is a noncrossing partition of [2k] with $k$ blocks of size two. There are $\left(\begin{array}{c}n \\ 2 k\end{array}\right)$ choices for $S$ and $\mathcal{C}_{k}$ choices for $\Lambda$ (see [35, Exercise 6.19o]).

To state the main theorem of this section, we require one last piece of notation. Define $\operatorname{Cov}(\Lambda)$ for a set partition $\Lambda$ to be the set of $\operatorname{arcs}(i, j) \in \operatorname{Arc}(\Lambda)$ with $j=i+1$; in other words,

$$
\operatorname{Cov}(\Lambda)=\operatorname{Arc}(\Lambda) \cap\{(i, i+1): i \in \mathbb{Z}\}
$$

Fix two additive abelian groups $\mathbb{A}$ and $\mathbb{B}$, and let $\Pi(n, \mathbb{A}, \mathbb{B})$ denote the set of labeled partitions $\Lambda \in \Pi(n, \mathbb{A} \oplus \mathbb{B})$ with

$$
\Lambda_{i j} \in \begin{cases}\mathbb{A} \backslash\{0\}, & \text { if }(i, j) \in \operatorname{Arc}(\Lambda) \backslash \operatorname{Cov}(\Lambda), \\ \mathbb{B} \backslash\{0\}, & \text { if }(i, j) \in \operatorname{Cov}(\Lambda) .\end{cases}
$$

We define $\mathrm{NC}(n, \mathbb{A}, \mathbb{B})$ analogously, as the set of noncrossing elements of $\Pi(n, \mathbb{A}, \mathbb{B})$. Note that the group $\mathrm{L}(n, \mathbb{B})$ acts on these sets by + , and that we may view shift as a map $\Pi(n, \mathbb{A}) \rightarrow \Pi(n+1, \mathbb{A}, \mathbb{B})$.

When $|\mathbb{A}|=x+1$ and $|\mathbb{B}|=y+1$, the cardinalities of $\Pi(n, \mathbb{A}, \mathbb{B})$ and $\mathrm{NC}(n, \mathbb{A}, \mathbb{B})$ are given respectively by the polynomials

$\mathcal{B}_{n}(x, y):=\sum_{\Lambda \in \Pi(n)} x^{|\operatorname{Arc}(\Lambda) \backslash \operatorname{Cov}(\Lambda)|} y^{|\operatorname{Cov}(\Lambda)|} \quad$ and $\quad \mathcal{N}_{n}(x, y):=\sum_{\Lambda \in \operatorname{NC}(n)} x^{|\operatorname{Arc}(\Lambda) \backslash \operatorname{Cov}(\Lambda)|} y^{|\operatorname{Cov}(\Lambda)|}$. 
We will derive more explicit expressions for these functions in a moment. In the mean time, let $\mathcal{B}_{n}(x):=\mathcal{B}_{n}(x, x)$ and $\mathcal{N}_{n}(x):=\mathcal{N}_{n}(x, x)$. These simpler polynomials have the formulas

$$
\mathcal{B}_{n}(x)=\sum_{k=0}^{n}\left\{\begin{array}{l}
n \\
k
\end{array}\right\} x^{n-k} \quad \text { and } \quad \mathcal{N}_{n}(x)=\sum_{k=0}^{n} N(n, k) x^{n-k},
$$

where $\left\{\begin{array}{l}n \\ k\end{array}\right\}$ and $N(n, k)$ are the Stirling numbers of the second kind and the Narayana numbers, defined as the number of ordinary and noncrossing set partitions of $[n]$ with $k$ blocks (equivalently, with $n-k$ arcs). We note the well-known formula $N(n, k)=$ $\frac{1}{n}\left(\begin{array}{l}n \\ k\end{array}\right)\left(\begin{array}{c}n \\ k-1\end{array}\right)$ for $n>0$ and adopt the convention $\left\{\begin{array}{l}0 \\ k\end{array}\right\}=N(0, k)=\delta_{k}$.

Remarks. Of course, $\mathcal{B}_{n}(1)$ is the $n$th Bell number and $\mathcal{N}_{n}(x)$ is the Narayana polynomial, whose values give the Catalan numbers when $x=1$ and the little Schröder numbers when $x=2$. The polynomials $\mathcal{B}_{n}(x)$ and $\mathcal{N}_{n}(x)$ are by definition the rank generating functions of the graded lattices $\Pi(n)$ and $\mathrm{NC}(n)$, though they have several alternate interpretations:

(i) As noted in [10, 18], when $x$ is a positive integer, $\left\{\mathcal{B}_{n}(x)\right\}_{n=0}^{\infty}$ and $\left\{\mathcal{N}_{n}(x)\right\}_{n=0}^{\infty}$ are the unique sequences respectively fixed by the operators

$$
\mathrm{R} \circ \underbrace{\text { BINOMIAL } \circ \cdots \circ \mathrm{BINOMIAL}}_{x \text { times }} \text { and } \mathrm{R} \circ \underbrace{\text { INVERT } \cdots \cdots \circ \text { INVERT, }}_{x \text { times }}
$$

where $\mathrm{R}\left(a_{0}, a_{1}, a_{2}, \ldots\right):=\left(1, a_{0}, a_{1}, a_{2}, \ldots\right)$ and BINOMIAL and INVERT are the sequence operators defined in [10].

(ii) As mentioned in the introduction, the $\mathbb{F}_{q}$-labeled partitions of $[n]$ index the supercharacters of the unitriangular group $\mathbf{U}_{n}\left(\mathbb{F}_{q}\right)$. There are $\mathcal{B}_{n}(q-1)$ distinct supercharacters of $\mathbf{U}_{n}\left(\mathbb{F}_{q}\right)$, of which $\mathcal{N}_{n}(q-1)$ are irreducible; see Section 7 .

(iii) If $G$ is a finite group then $\mathcal{B}_{2 k}(|G|)$ is the dimension of the $G$-colored partition algebra $P_{k}(x ; G)$ defined in [11] and studied (in the case $G=\mathbb{Z} / r \mathbb{Z}$ ) in [28].

The following theorem shows that $\mathcal{B}_{n}(x)$ counts the $\mathrm{L}(n+1, \mathbb{B})$-orbits in $\Pi(n+1, \mathbb{A}, \mathbb{B})$ while $\mathcal{M}_{n}(x)$ counts the $\mathrm{L}(n+1, \mathbb{B})$-orbits in $\mathrm{NC}(n+1, \mathbb{A}, \mathbb{B})$.

Theorem 3.3. For $n \geq 1$, the correspondence $\Lambda \mapsto\{\alpha+\operatorname{shift}(\Lambda): \alpha \in \mathrm{L}(n, \mathbb{B})\}$ is a bijection

$$
\begin{aligned}
\Pi(n-1, \mathbb{A}) & \rightarrow\{\mathrm{L}(n, \mathbb{B}) \text {-orbits in } \Pi(n, \mathbb{A}, \mathbb{B})\}, \\
\{\text { Poor elements of } \mathrm{NC}(n-1, \mathbb{A})\} & \rightarrow\{\mathrm{L}(n, \mathbb{B}) \text {-orbits in } \mathrm{NC}(n, \mathbb{A}, \mathbb{B})\} .
\end{aligned}
$$

Furthermore, the cardinality of the $\mathrm{L}(n, \mathbb{B})$-orbit of $\operatorname{shift}(\Lambda)$ is $|\mathbb{B}|^{s}$, where $s$ is the number of singleton blocks of $\Lambda \in \Pi(n-1, \mathbb{A})$. 
Proof. It is clear from our discussion of the action + that each $\mathrm{L}(n, \mathbb{B})$-orbit in $\Pi(n, \mathbb{A}, \mathbb{B})$ contains a unique 2-regular element and which is consequently of the form $\operatorname{shift}(\Gamma)$ for a unique $\Gamma \in \Pi(n-1, \mathbb{A})$. This shows that the first map is a bijection; the second map is also because $\operatorname{shift}(\Gamma)$ is noncrossing if and only if $\Gamma$ is poor and noncrossing, as a consequence of Lemma 3.1.

If $\Lambda=\operatorname{shift}(\Gamma)$, then $\operatorname{Arc}(\alpha) \cap \operatorname{Arc}(\Lambda)=\varnothing$ for all $\alpha \in \mathrm{L}(n, \mathbb{B})$, and one sees directly from Definition 1.1 that the $\mathrm{L}(n, \mathbb{B})$-orbit of $\Lambda$ has size $|\mathbb{B}|^{s}$ where $s$ is the number of superdiagonal hooks $\{(i, j+1): i<j\} \cup\{(j, k): j<k\}$ for $j \in[n-1]$ which contain no nonzero entries in the matrix of $\Lambda$. Consulting the definition of shift, we find that the hook containing $(j, j+1)$ belongs to this set if and only if $\{j\}$ is a singleton block of $\Gamma$.

The following theorem uses the previous result to derive two equivalent formulas for each of the polynomials $\mathcal{B}_{n}(x, y)$ and $\mathcal{N}_{n}(x, y)$.

Theorem 3.4. If $n$ is a nonnegative integer then the following identities hold:

$$
\begin{aligned}
& \text { (1) } \mathcal{B}_{n+1}(x, y)=\sum_{k=0}^{n}\left(\begin{array}{l}
n \\
k
\end{array}\right) \mathcal{B}_{k}(x) y^{n-k}=\sum_{k=0}^{n}\left(\begin{array}{l}
n \\
k
\end{array}\right) \mathcal{F}_{k}(x)(y+1)^{n-k} . \\
& \text { (2) } \mathcal{N}_{n+1}(x, y)=\sum_{k=0}^{n}\left(\begin{array}{l}
n \\
k
\end{array}\right) \mathcal{M}_{k}(x) y^{n-k}=\sum_{k=0}^{\lfloor n / 2\rfloor} \mathcal{C}_{k}\left(\begin{array}{c}
n \\
2 k
\end{array}\right) x^{k}(y+1)^{n-2 k} .
\end{aligned}
$$

Proof. In each part, the terms in first sum counts partitions whose matrices have $n-k$ nonzero entries on the superdiagonal $\{(i, i+1): i \in[n]\}$, while the terms in the second sum count partitions whose $\mathrm{L}(n+1, \mathbb{B})$-orbits have the same fixed size. Thus the sums in each part are necessarily equal to each other and to $\mathcal{B}_{n+1}(x, y)$ in $(1)$ and $\mathcal{N}_{n+1}(x, y)$ in (2).

In detail, $\left(\begin{array}{l}n \\ k\end{array}\right) \mathcal{B}_{k}(x) y^{n-k}$ is the number of elements of $\Pi(n+1, \mathbb{A}, \mathbb{B})$ whose matrices have $n-k$ nonzero entries on the superdiagonal since there are $\left(\begin{array}{l}n \\ k\end{array}\right) y^{n-k}$ choices for the entries and their positions, and since deleting the rows and columns containing these positions produces the matrix of $\operatorname{shift}(\Lambda)$ for an arbitrary $\Lambda \in \Pi(k, \mathbb{A})$. On the other hand, $\left(\begin{array}{l}n \\ k\end{array}\right) \mathcal{F}_{k}(x)(y+1)^{n-k}$ is the number of elements of $\Pi(n+1, \mathbb{A}, \mathbb{B})$ whose $\mathrm{L}(n+1, \mathbb{B})$ orbits have size $(y+1)^{n-k}$ by Theorem 3.3 , since there are $\left(\begin{array}{l}n \\ k\end{array}\right) \mathcal{F}_{k}(x)$ distinct $\mathbb{A}$-labeled set partitions of $[n]$ with $n-k$ singleton blocks.

Likewise, $\left(\begin{array}{l}n \\ k\end{array}\right) \mathcal{M}_{k}(x) y^{n-k}$ is the number of elements of $\mathrm{NC}(n+1, \mathbb{A}, \mathbb{B})$ whose matrices have $n-k$ nonzero entries on the superdiagonal since deleting the rows and columns containing these positions produces the matrix of shift $(\Lambda)$ for an arbitrary poor $\Lambda \in$ $\mathrm{NC}(k, \mathbb{A})$. By Theorem 3.3, the number of elements of $\mathrm{NC}(n+1, \mathbb{A}, \mathbb{B})$ whose $\mathrm{L}(n+1, \mathbb{B})$ orbits have size $(y+1)^{n-k}$ is equal to the number of poor elements of $\mathrm{NC}(n, \mathbb{A})$ with $n-k$ singleton blocks, which is the product of $\left(\begin{array}{c}n \\ n-k\end{array}\right)$ with the number of partitions in $\mathrm{NC}(k, \mathbb{A})$ whose blocks all have size two. The latter number is clearly 0 if $k$ is odd, and is equal to $x^{k / 2}$ times the leading coefficient of $\mathcal{M}_{k}(x)$ if $k$ is even.

Remarks. Both parts of the theorem deserve a few comments. 
(i) Setting $y=0$ in the first part shows that $\mathcal{B}_{n}(x)$ is the binomial transform of $\mathcal{F}_{n}(x)$; i.e., $\mathcal{B}_{n}(x)=\sum_{k}\left(\begin{array}{l}n \\ k\end{array}\right) \mathcal{F}_{k}(x)$ (see [27, Lemma 5.2] for a combinatorial proof). Setting $x=y$ in (1) yields $\mathcal{B}_{n+1}(x)=\sum_{k=0}^{n}\left(\begin{array}{l}n \\ k\end{array}\right) \mathcal{B}_{k}(x) x^{n-k}$, an identity noted several places previously [24, 31], which is equivalent to the standard recurrence formula for the Touchard polynomials.

(ii) We may rewrite the second part as the equation

$$
\sum_{i=0}^{\lfloor n / 2\rfloor} \sum_{j=0}^{n-2 i} \frac{1}{n+1}\left(\begin{array}{c}
n+1 \\
i+1
\end{array}\right)\left(\begin{array}{c}
n-i \\
j
\end{array}\right)\left(\begin{array}{c}
n-i-j \\
i
\end{array}\right) x^{i} y^{j}=\sum_{k=0}^{\lfloor n / 2\rfloor} \mathcal{C}_{k}\left(\begin{array}{c}
n \\
2 k
\end{array}\right) x^{k}(y+1)^{n-2 k} .
$$

Chen, Deutsch, and Elizalde give a combinatorial proof of this identity using a correspondence between plane trees and 2-Motzkin paths [14, Theorem 9]. Setting $x=y$ in part (2) produces Coker's identity (1.2) mentioned in the introduction and taking $x=y=1$ recovers Touchard's classical identity $\mathcal{C}_{n+1}=\sum_{k} \mathcal{C}_{k}\left(\begin{array}{c}n \\ 2 k\end{array}\right) 2^{n-2 k}$.

As one consequence of the theorem, we employ the inclusion-exclusion principle to compute alternate formulas counting the invariant elements in $\Pi(n, \mathbb{A}, \mathbb{B})$ and $\mathrm{NC}(n, \mathbb{A}, \mathbb{B})$.

Corollary 3.5. For each integer $n \geq 0$, the following identities hold:

(1) $\sum_{k=0}^{n}(-1)^{n-k}\left(\begin{array}{l}n \\ k\end{array}\right)(y+1)^{n-k} \mathcal{B}_{k+1}(x, y)=\mathcal{F}_{n}(x)$.

(2) $\sum_{k=0}^{n}(-1)^{n-k}\left(\begin{array}{l}n \\ k\end{array}\right)(y+1)^{n-k} \mathcal{N}_{k+1}(x, y)= \begin{cases}x^{n / 2} \mathcal{C}_{n / 2}, & \text { if } n \text { is even, } \\ 0, & \text { if } n \text { is odd. }\end{cases}$

Remark. Setting $x=y$ in part (2) recovers one of the three identities given by Mansour and Sun in [26, Theorem 1.1]. This equation with $x=y=1$ appeared earlier as [12, Proposition 2.2] and has been studied in a number of places; Chen, Wang, and Zhao provide a nice bibliography in the discussion preceding [15, Theorem 2.4].

Proof. Given a subset $S \subset[n]$, let $\mathcal{X}_{S}$ and $\mathcal{Y}_{S}$ denote the unions of the $\mathrm{L}(n, \mathbb{B})$-orbits of $\operatorname{shift}(\Lambda)$ for all partitions $\Lambda$ in $\Pi(n, \mathbb{A})$ and $\mathrm{NC}(n, \mathbb{A})$, respectively, which contain the singleton $\{i\}$ as a block for each $i \in S$. It is straightforward to compute from Theorems 3.3 and 3.4 that

$$
\left|\mathcal{X}_{S}\right|=(y+1)^{|S|} \mathcal{B}_{n-|S|+1}(x, y) \quad \text { and } \quad\left|\mathcal{Y}_{S}\right|=(y+1)^{|S|} \mathcal{N}_{n-|S|+1}(x, y) .
$$

The inclusion-exclusion principle now affords our result since by Theorem 3.3 the sets of $\mathrm{L}(n, \mathbb{B})$-invariants in $\Pi(n+1, \mathbb{A}, \mathbb{B})$ and $\mathrm{NC}(n+1, \mathbb{A}, \mathbb{B})$ are the respective complements of $\bigcup_{i \in[n]} \mathcal{X}_{\{i\}}$ and $\bigcup_{i \in[n]} \mathcal{Y}_{\{i\}}$. 


\section{A short digression on nonnesting partitions}

In the next section we introduce type $\mathrm{B}$ and $\mathrm{D}$ analogues for the sets $\Pi(n, \mathbb{A})$ and $\mathrm{NC}(n, \mathbb{A})$. Before studying these new families of set partitions, it will be useful to prepare the way with some requisite notation.

To this end, we recall that a set partition $\Lambda$ is nonnesting if no two $\operatorname{arcs}(i, l),(j, k) \in$ $\operatorname{Arc}(\Lambda)$ have $i<j<k<l$. Visually, this means that no arc "nests" beneath another in $\Lambda$ 's standard representation. Let $[ \pm n]=\{ \pm 1, \pm 2, \ldots, \pm n\}$ and write $-\Lambda$ for the set partition whose blocks are $-B$ for $B \in \Lambda$. Now define

$$
\begin{aligned}
\mathrm{NN}(n) & :=\text { the set of nonnesting partitions of }[n] \\
\mathrm{NN}^{\mathrm{B}}(n) & :=\text { the set of nonnesting partitions } \Lambda \text { of }[ \pm n] \text { with } \Lambda=-\Lambda
\end{aligned}
$$

The elements of $\mathrm{NN}(n+1)$ and $\mathrm{NN}^{\mathrm{B}}(n)$ can be viewed as the type $A_{n}$ and $B_{n}$ instances of a more general object called a "nonnesting partition" with many interesting properties. We direct the reader to [5, 6] for a more detailed exposition, as here we shall only discuss a few basic facts.

To begin, there is a close relationship between $\mathrm{NN}(n)$ and $\mathrm{NC}(n)$ : the number of nonnesting and noncrossing set partitions of $[n]$ are equidistributed by type $[6$, Theorem 3.1 , where the type of a set partition is the partition of the number $n$ whose parts are the sizes of the set partition's blocks. The following simple bijection uncross : $\mathrm{NN}(n) \rightarrow$ $\mathrm{NC}(n)$ is not type-preserving but will be of some use later. We define this by the algorithm below (see also [5, Section 5.1]):

1. Given any set partition $\Lambda$, let $A=\operatorname{Arc}(\Lambda)$.

2. While $A$ has at least one pair of crossing arcs: choose two $\operatorname{arcs}(i, k),(j, l) \in A$ with $i<j<k<l$ and replace $A$ with $(A-\{(i, k),(j, l)\}) \cup\{(i, l),(j, k)\}$.

3. Define $\operatorname{uncross}(\Lambda)$ as the noncrossing set partition of $[n]$ with arc set $A$.

Note that this algorithm makes sense for any partition of a finite set of integers. The procedure locally converts each crossing to a nesting in the standard representation of $\Lambda$; i.e.,

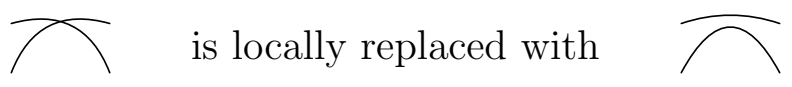

so that, e.g., uncross $(14|25| 36)=16|25| 34$ and uncross $(\Lambda)=\Gamma$ in $(1.1)$. This observation makes clear that the algorithm's output has no dependence on the order in which the pairs of crossing arcs are chosen in the second step. Thus uncross gives a well-defined map $\Pi(n) \rightarrow \mathrm{NC}(n)$, the important properties of which are the following:

- $\operatorname{uncross}(\Lambda)$ has the same number of blocks as $\Lambda$.

- uncross defines a bijection from $\mathrm{NN}(n) \rightarrow \mathrm{NC}(n)$.

- uncross defines a bijection $\mathrm{NN}^{\mathrm{B}}(n) \rightarrow\{$ Noncrossing $\Lambda \vdash[ \pm n]$ with $\Lambda=-\Lambda\}$. 
The first property is clear since uncross $(\Lambda)$ has the same number of $\operatorname{arcs}$ as $\Lambda$; the second property is well-known; and the third follows from the second since uncross $(-\Lambda)=$ $-\operatorname{uncross}(\Lambda)$.

We see from this discussion that $\mathrm{NN}(n)$ has $N(n, k)$ elements with $k$ blocks and $\mathcal{C}_{n}$ elements in total. The following observation lists analogous statistics for $\mathrm{NN}^{\mathrm{B}}(n)$.

Lemma 4.1. $\mathrm{NN}^{\mathrm{B}}(n)$ has $\left(\begin{array}{l}n \\ k\end{array}\right)^{2}$ elements with $2 k$ or $2 k+1$ blocks, and $\left(\begin{array}{c}2 n \\ n\end{array}\right)$ elements in total.

Proof. The involution on set partitions of $[ \pm n]$ induced by the map

$$
i \mapsto \begin{cases}i-n-1, & \text { if } i \in[n] \\ i+n+1, & \text { if }-i \in[n]\end{cases}
$$

gives a bijection from $\mathrm{NN}^{\mathrm{B}}(n)$ to what Athanasiadis [6] calls the $B_{n}$-partitions associated to the nonnesting partitions of type $B_{n}$. Hence the lemma is simply a restatement of [6, Corollary 5.8].

Recall that a Dyck path with $2 n$ steps is a lattice path beginning at $(0,0)$ and ending at $(n, 0)$ which uses only the steps $(1,1)$ and $(1,-1)$ and which never travels below the $y$-axis. It is well-known that the set $\mathcal{D}_{n}$ of Dyck paths with $2 n$ steps has cardinality $\left|\mathcal{D}_{n}\right|=|\mathrm{NC}(n)|=|\mathrm{NN}(n)|=\mathcal{C}_{n}$, and there is an especially simple bijection $\mathrm{NN}(n) \rightarrow \mathcal{D}_{n}$. Namely, we associate to a nonnesting set partition $\Lambda \in \mathrm{NN}(n)$ the unique path in $\mathcal{D}_{n}$ whose valleys (the points which simultaneously end a downstep $(1,-1)$ and begin an upstep $(1,1))$ are the points $(j+i-1, j-i-1)$ for $(i, j) \in \operatorname{Arc}(\Lambda)$. Intuitively, this is the path tracing the upper boundary of the squares in the matrix of $\Lambda$ which are south or west of nonzero entries.

Call a Dyck path with $2 n$ steps symmetric if the path is symmetric about the vertical line $x=n$. The order-preserving bijection $[ \pm n] \rightarrow[2 n]$ induces an inclusion of $\mathrm{NN}^{\mathrm{B}}(n)$ in $\mathrm{NN}(2 n)$, and it clear that with respect to this identification, the bijection $\mathrm{NN}(2 n) \rightarrow \mathcal{D}_{2 n}$ just mentioned restricts to a bijection from $\mathrm{NN}^{\mathrm{B}}(n)$ to the set of symmetric Dyck paths with $4 n$ steps. Hence:

Lemma 4.2. There are $\left(\begin{array}{c}2 n \\ n\end{array}\right)$ symmetric Dyck paths with $4 n$ steps.

\section{Analogues in other types}

We are now prepared to discuss two analogues for our "classical" notion of a labeled set partition. In detail, given a nonnegative integer and an additive abelian group, we define $\Pi^{\mathrm{B}}(n, \mathbb{A})$ (respectively, $\left.\Pi^{\mathrm{D}}(n, \mathbb{A})\right)$ as the set of $\mathbb{A}$-labeled set partitions $\Lambda$ of $\{0\} \cup[ \pm n]$ (respectively, $[ \pm n]$ ) with the property that

$$
(i, j) \in \operatorname{Arc}(\Lambda) \quad \text { if and only if } \quad(-j,-i) \in \operatorname{Arc}(\Lambda) \text { and } \Lambda_{i j}+\Lambda_{-j,-i}=0 .
$$


We write $\Pi^{\mathrm{B}}(n):=\Pi^{\mathrm{B}}\left(n, \mathbb{F}_{2}\right)$ and $\Pi^{\mathrm{D}}(n)=\Pi^{\mathrm{D}}\left(n, \mathbb{F}_{2}\right)$ for the corresponding sets of unlabeled set partitions.

The condition (5.1) implies that $\Lambda$ has no arcs of the form $(-i, i)$ and hence that $|\operatorname{Arc}(\Lambda)|$ is even, and that if $B$ is a block of $\Lambda$ then $-B$ is also a block. If $\Lambda \in \Pi^{\mathrm{B}}(n, \mathbb{A})$ then exactly one block $B \in \Lambda$ contains zero and has $B=-B$, while if $\Lambda \in \Pi^{\mathrm{D}}(n, \mathbb{A})$ then every block $B \in \Lambda$ has $B \neq-B$. For example, the elements of $\Pi^{\mathrm{B}}(2, \mathbb{A})$ are
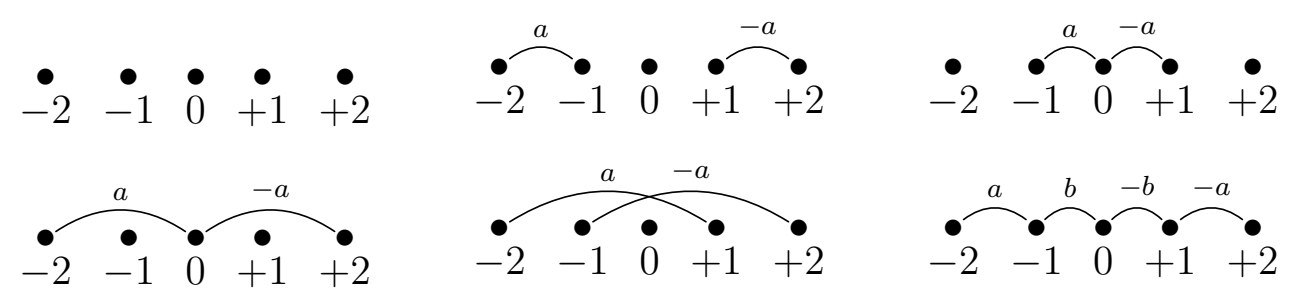

and the elements of $\Pi^{\mathrm{D}}(2, \mathbb{A})$ are
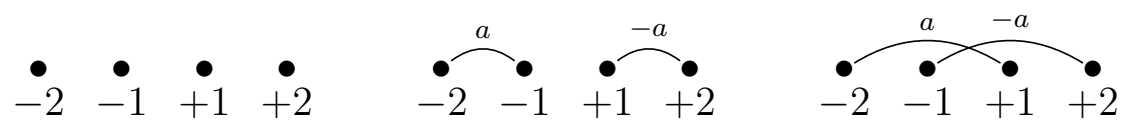

for $a, b \in \mathbb{A} \backslash\{0\}$.

We have three reasons to suggest these sets as the type $\mathrm{B}$ and $\mathrm{D}$ analogues of $\Pi(n, \mathbb{A})$. First and possibly foremost, $\Pi^{\mathrm{B}}\left(n, \mathbb{F}_{q}\right)$ and $\Pi^{\mathrm{D}}\left(n, \mathbb{F}_{q}\right)$ are the natural indexing sets for the supercharacters defined in [1, 2, 3] of the the Sylow $p$-subgroups of the Chevalley groups of type $B_{n}$ and $D_{n}$ over $\mathbb{F}_{q}$ (where $p$ is the odd characteristic of $\mathbb{F}_{q}$ ); see Section 7 below for an explicit definition. Thus, in analogy with our techniques in Section 3, we can use the multiplicative action of the linear characters of these groups on their supercharacters to define a combinatorial action of a subset of $\Pi^{\mathrm{B}}(n, \mathbb{A})$ or $\Pi^{\mathrm{D}}(n, \mathbb{A})$ on itself. From an analysis of the orbits of this action, we may then attempt to derive identities in the style of Theorem 3.4 .

The papers [1, 2, 3] also define a set of supercharacters for the Sylow $p$-subgroups of the finite Chevalley groups of type $C_{n}$, which should motivate the definition of a third family of labeled set partitions $\Pi^{\mathrm{C}}(n, \mathbb{A})$. We omit this family from the present work because its investigation fits less naturally into our exposition and seems not to yield any really new identities.

A second explanation for our notation comes from the following observation. The order-preserving bijections $\{0\} \cup[ \pm n] \rightarrow[2 n+1]$ and $[ \pm n] \rightarrow[2 n]$ induce inclusions $\Pi^{\mathrm{B}}(n, \mathbb{A}) \hookrightarrow \Pi(2 n+1, \mathbb{A})$ and $\Pi^{\mathrm{D}}(n, \mathbb{A}) \hookrightarrow \Pi(2 n, \mathbb{A})$, and we define the matrix of $\Lambda$ in $\Pi^{\mathrm{B}}(n, \mathbb{A})$ or $\Pi^{\mathrm{D}}(n, \mathbb{A})$ to be the matrix of the corresponding set partition in $\Pi(2 n+1, \mathbb{A})$ or $\Pi(2 n, \mathbb{A})$. If $\mathbb{C}$ is the field of complex numbers and

$$
\mathfrak{s o}^{\prime}(n, \mathbb{C}):=\left\{X \in \mathfrak{s l}(n, \mathbb{C}): X+X^{\dagger}=0\right\}, \quad \text { where }\left(X^{\dagger}\right)_{i j}:=X_{n+1-j, n+1-i},
$$

then the map assigning a set partition to its matrix gives a bijection from $\Pi(n+1, \mathbb{C})$, $\Pi^{\mathrm{B}}(n, \mathbb{C})$, and $\Pi^{\mathrm{D}}(n, \mathbb{C})$ to the sets of strictly upper triangular matrices with at most one 
nonzero entry in each row and column in $\mathfrak{s l}(n+1, \mathbb{C}), \mathfrak{s o}^{\prime}(2 n+1, \mathbb{C})$, and $\mathfrak{s o}^{\prime}(2 n, \mathbb{C})$, which we may regard as the complex simple Lie algebras of types $A_{n}, B_{n}$, and $D_{n}$.

Finally, we mention that the unlabeled partitions $\Pi(n+1)$ and $\Pi^{\mathrm{B}}(n)$ are naturally identified with the intersection lattice of the Coxeter hyperplane arrangements of type $A_{n}$ and $B_{n}$ (see [29]). The set $\Pi^{\mathrm{D}}(n)$ is not similarly related to the intersection lattice of the type $D_{n}$ Coxeter hyperplane arrangement, however.

The sets of noncrossing elements of $\Pi^{\mathrm{B}}(n, \mathbb{A})$ and $\Pi^{\mathrm{D}}(n+1, \mathbb{A})$ are both in bijection with $\mathrm{NC}(n, \mathbb{A})$, so it will be fruitful to introduce a different kind of "noncrossing" partition to investigate. To this end, for $\mathrm{X} \in\{\mathrm{B}, \mathrm{D}\}$, we let $\widetilde{\mathrm{NC}}^{\mathrm{X}}(n, \mathbb{A})$ denote the subset of $\Pi^{\mathrm{X}}(n, \mathbb{A})$ consisting of $\mathbb{A}$-labeled set partitions $\Lambda$ with the following "noncrossing" property:

If there are $(i, k),(j, l) \in \operatorname{Arc}(\Lambda)$ such that $i<j<k<l$ then $(i, k)=(-l,-j)$.

As usual, we write $\widetilde{\mathrm{NC}^{\mathrm{X}}}(n):=\widetilde{\mathrm{NC}}^{\mathrm{X}}\left(n, \mathbb{F}_{2}\right)$ to indicate the corresponding sets of unlabeled set partitions. This set generalizes $\mathrm{NC}(n, \mathbb{A})$ in the following sense: one may define $\mathrm{NC}(n, \mathbb{A})$ as the subset of $\Lambda \in \Pi(n, \mathbb{A})$ such that if $(i, k),(j, l) \in \operatorname{Arc}(\Lambda)$ and $\Gamma \in \Pi(n, \mathbb{A})$ then $\{(i, j),(j, k),(k, l)\} \not \subset \operatorname{Arc}(\Gamma)$. The same definition with $\Pi(n, \mathbb{A})$ replaced by $\Pi^{\mathrm{X}}(n, \mathbb{A})$ gives $\widetilde{\mathrm{NC}}^{\mathrm{X}}(n, \mathbb{A})$. Alternatively, Theorem 7.3 below provides a representation theoretic characterization of $\widetilde{N C}^{\mathrm{X}}(n, \mathbb{A})$ in terms of its associated set of supercharacters.

As with $\Pi(n)$, the sets $\Pi^{\mathrm{B}}(n)$ and $\Pi^{\mathrm{D}}(n)$ are partially ordered by refinement, and graded by the rank functions

$$
\operatorname{rank}(\Lambda):= \begin{cases}n-(|\Lambda|-1) / 2, & \text { for } \Lambda \in \Pi^{\mathrm{B}}(n), \\ n-|\Lambda| / 2, & \text { for } \Lambda \in \Pi^{\mathrm{D}}(n)\end{cases}
$$

Both $\Pi^{\mathrm{B}}(n)$ and $\Pi^{\mathrm{D}}(n)$ are meet semilattices since any collection of elements $\left\{\Lambda_{i}\right\}$ has a greatest lower bound given by the partition whose blocks are the nonempty intersections of the form $\bigcap_{i} B_{i}$ where each $B_{i} \in \Lambda_{i}$. However, of the two, only $\Pi^{\mathrm{B}}(n)$ possesses a greatest element and is therefore a lattice. The meet of any collection of elements in $\widetilde{N C}^{\mathrm{X}}(n)$ also lies in $\widetilde{\mathrm{NC}^{\mathrm{X}}}(n)$ for $\mathrm{X} \in\{\mathrm{B}, \mathrm{D}\}$, and it follows that $\widetilde{\mathrm{NC}^{\mathrm{B}}}(n)$ is likewise a graded lattice (with height $n$ ) while $\widetilde{\mathrm{NC}^{\mathrm{D}}}(n)$ is only a graded meet semilattice (with height $n-1$ ).

Remark. To any Coxeter system $(W, S)$ there corresponds a lattice of noncrossing partitions, defined as the interval between the identity and any fixed Coxeter element in the absolute order of $W$. There is a large body of literature on this subject; see [5] for a useful survey. The noncrossing partition lattice of the Coxeter system of type $A_{n-1}$ coincides with $\mathrm{NC}(n)$, and the lattices of types $B_{n}$ and $D_{n}$, which we might as well denote by $\mathrm{NC}^{\mathrm{B}}(n)$ and $\mathrm{NC}^{\mathrm{D}}(n)$, may be realized combinatorially as subposets of $\Pi^{\mathrm{B}}(n)$. However, $\mathrm{NC}^{\mathrm{B}}(n)$ and $\mathrm{NC}^{\mathrm{D}}(n)$ are neither obviously related to $\widetilde{\mathrm{NC}^{\mathrm{B}}}(n)$ and $\widetilde{\mathrm{NC}^{\mathrm{D}}}(n)$ (though there are connections between them), nor preserved by the group action defined below, so the somewhat more obscure sets $\widetilde{N C}^{\mathrm{B}}(n)$ and $\widetilde{\mathrm{NC}}^{\mathrm{D}}(n)$ are better suited to our purposes. 
Write $\mathrm{L}^{\mathrm{X}}(n, \mathbb{A})$ to denote the type $\mathrm{X}$ analogue of $\mathrm{L}(n, \mathbb{A})$ : viz., the set of labeled partitions $\Lambda \in \Pi^{\mathrm{X}}(n, \mathbb{A})$ whose blocks consist of consecutive integers or, equivalently, which have $\operatorname{Arc}(\Lambda)=\operatorname{Cov}(\Lambda)$. We define $\alpha+\Lambda$ for $\alpha \in \mathrm{L}^{\mathrm{X}}(n, \mathbb{A})$ and $\Lambda \in \Pi^{\mathrm{X}}(n, \mathbb{A})$ exactly as in Definition 1.1 , only with $\mathrm{L}(n, \mathbb{A})$ and $\Pi(n, \mathbb{A})$ replaced by $\mathrm{L}^{\mathrm{X}}(n, \mathbb{A})$ and $\Pi^{\mathrm{X}}(n, \mathbb{A})$. For example, if

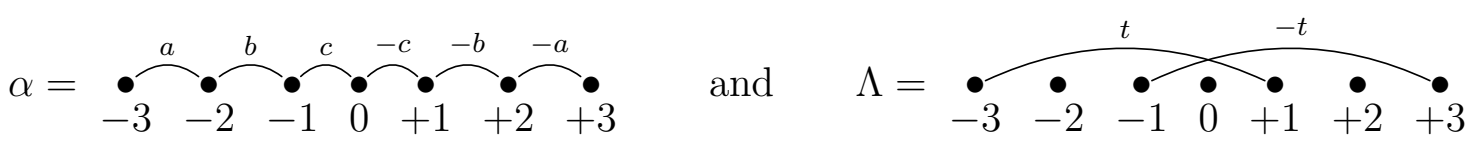

for $a, b, c, d, e, t \in \mathbb{A} \backslash\{0\}$ then we have

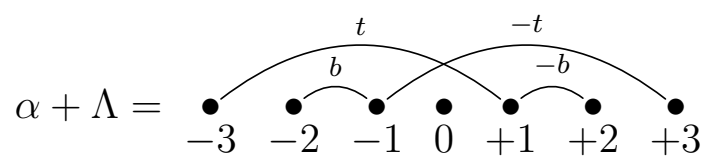

Note that we may alternately characterize $\alpha+\Lambda$ as in Observation 2.1, or in terms of the matrices of $\alpha \in \mathrm{L}^{\mathrm{X}}(n, \mathbb{A})$ and $\Lambda \in \Pi^{\mathrm{X}}(n, \mathbb{A})$ as in Observation 2.2. As before, the operation + makes $\mathrm{L}^{\mathrm{X}}(n, \mathbb{A})$ into an abelian group (isomorphic to $\mathbb{A}^{n}$ if $\mathrm{X}=\mathrm{B}$ and to $\mathbb{A}^{n-1}$ if $\left.\mathrm{X}=\mathrm{D}\right)$ acting on $\Pi^{\mathrm{X}}(n, \mathbb{A})$, and it is evident from our definitions that this action preserves $\widetilde{\mathrm{NC}^{\mathrm{X}}}(n, \mathbb{A})$.

Recall the definition in Section 2 of $\Lambda^{+}$for an arbitrary set partition $\Lambda$. If we view unlabeled set partitions as $\mathbb{F}_{2}$-labeled, then this definition amounts to the formulas

$$
\begin{array}{ll}
\Lambda^{+}=\{\{-n, \ldots,-1,0,1, \ldots, n\}\}+\Lambda, & \text { for } \Lambda \in \Pi^{\mathrm{B}}(n), \\
\Lambda^{+}=\{\{-n, \ldots,-1\},\{1, \ldots, n\}\}+\Lambda, & \text { for } \Lambda \in \Pi^{\mathrm{D}}(n) .
\end{array}
$$

Hence $\Lambda \mapsto \Lambda^{+}$defines an involution of both $\Pi^{\mathrm{X}}(n)$ and $\widetilde{\mathrm{NC}^{\mathrm{X}}}(n)$ (as sets, not lattices) for $\mathrm{X} \in\{\mathrm{B}, \mathrm{D}\}$. The following analogue of Proposition 2.4 uses this involution to show that the lattice $\widetilde{\mathrm{NC}}^{\mathrm{B}}(n)$ is rank symmetric.

Proposition 5.1. The map $\Lambda \mapsto \Lambda^{+}$is rank inverting on $\widetilde{\mathrm{NC}^{\mathrm{B}}}(n)$. That is, if $\Lambda \in \widetilde{\mathrm{NC}^{\mathrm{B}}}(n)$ has $2 k+1$ blocks, then $\Lambda^{+} \in \widetilde{\mathrm{NC}}^{\mathrm{B}}(n)$ has $2(n-k)+1$ blocks.

Remark. The lattice of type $B_{n}$ noncrossing partitions is also rank symmetric; in fact, it is self-dual and locally self-dual [5, 29]. The lattice $\widetilde{\mathrm{NC}}^{\mathrm{B}}(n)$ fails to possess any of these stronger properties when $n \geq 4$.

Proof. Observe that the definition of uncross $(\Lambda)$ make sense for $\Lambda \in \Pi^{\mathrm{B}}(n)$, although the set partition uncross $(\Lambda) \vdash\{0\} \cup[ \pm n]$ may no longer belong to $\Pi^{\mathrm{B}}(n)$. It is straightforward to check that $\Lambda^{+} \in \widetilde{\mathrm{NC}^{\mathrm{B}}}(n)$ if $\Lambda \in \widetilde{\mathrm{NC}^{\mathrm{B}}}(n)$ and that $\Lambda$ and $\Lambda^{+}$have the same crossings, which are always pairs of arcs of the form $(-i, j),(-j, i)$ for $i, j \in[n]$. It follows that uncross $(\Lambda)^{+}=\operatorname{uncross}\left(\Lambda^{+}\right)$. Since uncross preserves the number of blocks in a set partition and since both uncross and the involution + commute with natural inclusion $\widetilde{N C}^{\mathrm{B}}(n) \hookrightarrow \Pi(2 n+1)$, our result is a consequence of Proposition 2.4. 
As a corollary, we similarly compute the number of blocks in $\Lambda^{+}$for $\Lambda \in \widetilde{\mathrm{NC}}^{\mathrm{D}}(n)$.

Corollary 5.2. If $\Lambda \in \widetilde{\mathrm{NC}}^{\mathrm{D}}(n)$ then

$$
\left|\Lambda^{+}\right|= \begin{cases}2 n+2-|\Lambda|, & \text { if }-1 \text { is the greatest element of its block in } \Lambda, \\ 2 n-|\Lambda|, & \text { otherwise. }\end{cases}
$$

Proof. Let $\varphi: \widetilde{\mathrm{NC}}^{\mathrm{D}}(n) \rightarrow \widetilde{\mathrm{NC}}^{\mathrm{B}}(n)$ be the injective map which adds the singleton block $\{0\}$ to $\Lambda \in \widetilde{N C}^{\mathrm{D}}(n)$; i.e., $\varphi(\Lambda)=\Lambda \cup\{\{0\}\}$. It is easy to see that if -1 is not the greatest element of its block in $\Lambda \in \widetilde{\mathrm{NC}^{\mathrm{D}}}(n)$, so that $\Lambda$ has arcs of the form $(-1, i),(-i, 1)$ for some $1<i \leq n$, then $\varphi\left(\Lambda^{+}\right)=\varphi(\Lambda)^{+}$. In this case $\left|\Lambda^{+}\right|=2 n+2-|\Lambda|$ by the previous proposition applied to $\varphi(\Lambda) \in \widetilde{\mathrm{NC}^{\mathrm{B}}}(n)$. On the other hand, if -1 is the greatest element of its block in $\Lambda$, then $\varphi(\Lambda)^{+}$is formed from $\varphi\left(\Lambda^{+}\right)$by adding the $\operatorname{arcs}(-1,0)$ and $(0,1)$. Hence $\varphi(\Lambda)^{+}$has two fewer blocks than $\varphi\left(\Lambda^{+}\right)$, so we now obtain $\left|\Lambda^{+}\right|=2 n-|\Lambda|$ from the previous proposition applied to $\varphi(\Lambda)$.

Fix two additive abelian groups $\mathbb{A}$ and $\mathbb{B}$ and define $\Pi^{\mathrm{X}}(n, \mathbb{A}, \mathbb{B})$ and $\widetilde{\mathrm{NC}}^{\mathrm{X}}(n, \mathbb{A}, \mathbb{B})$ for $\mathrm{X} \in\{\mathrm{B}, \mathrm{D}\}$ as the subsets of $\Pi^{\mathrm{X}}(n, \mathbb{A} \oplus \mathbb{B})$ and $\widetilde{\mathrm{NC}^{\mathrm{X}}}(n, \mathbb{A} \oplus \mathbb{B})$ consisting of labeled set partitions $\Lambda$ satisfying (3.1). Note as in Section 3 that + defines an action of $\mathrm{L}^{\mathrm{X}}(n, \mathbb{B})$ on $\Pi^{\mathrm{X}}(n, \mathbb{A}, \mathbb{B})$ and $\widetilde{\mathrm{NC}}^{\mathrm{x}}(n, \mathbb{A}, \mathbb{B})$. Mirroring our previous notation, we define polynomials

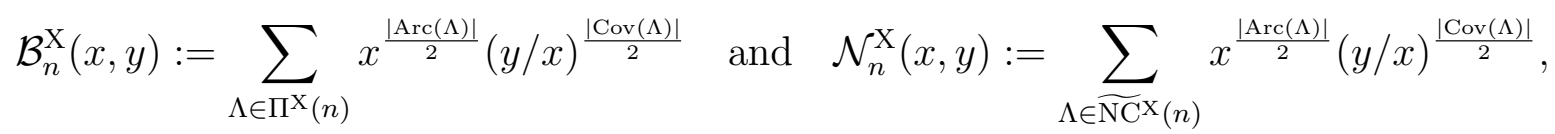

and let $\mathcal{B}_{n}^{\mathrm{X}}(x):=\mathcal{B}_{n}^{\mathrm{X}}(x, x)$ and $\mathcal{N}_{n}^{\mathrm{X}}(x):=\mathcal{N}_{n}^{\mathrm{X}}(x, x)$.

Clearly $\mathcal{B}_{n}^{\mathrm{B}}(x)=\sum_{k=0}^{n}\left\{\begin{array}{l}n \\ k\end{array}\right\}_{\mathrm{B}} x^{n-k}$ where $\left\{\begin{array}{l}n \\ k\end{array}\right\}_{\mathrm{B}}$ denotes the number of set partitions in $\Pi^{\mathrm{B}}(n)$ with $2 k+1$ blocks. The numbers $\left\{\begin{array}{l}n \\ k\end{array}\right\}_{\mathrm{B}}$ coincide with the Whitney numbers of the second kind $W_{2}(n, k)$ studied in [8, 9] and appear as sequence [33, A039755]. On the other hand, since the blocks of set partitions in $\Pi^{\mathrm{D}}(n)$ comes in pairs $\pm B$, the preimage of $\Lambda \in \Pi(n)$ under the surjection $\Pi^{\mathrm{D}}(n) \rightarrow \Pi(n)$ induced by the absolute value map $[ \pm n] \rightarrow[n]$ contains $2^{n-|\Lambda|}=2^{|\operatorname{Arc}(\Lambda)|}$ elements, all having $2|\operatorname{Arc}(\Lambda)| \operatorname{arcs}$. Hence

$$
\mathcal{B}_{n}^{\mathrm{D}}(x)=\mathcal{B}_{n}(2 x), \quad \text { for all } n \geq 0 .
$$

These formulas show that $\left\{\mathcal{B}_{n}^{\mathrm{B}}(1)\right\}_{n=0}^{\infty}=(1,2,6,24,116,648,4088, \ldots)$ gives the sequence of Dowling numbers [33, A007405] while $\left\{\mathcal{B}_{n}^{\mathrm{D}}(1)\right\}_{n=0}^{\infty}=(1,1,3,11,49,257,1539, \ldots)$ gives sequence [33, A004211].

The following result generalizes the recurrence $\mathcal{B}_{n}(x)=\sum_{k}\left(\begin{array}{l}n \\ k\end{array}\right) \mathcal{B}_{k}(x)$ noted in the first remark to Theorem 3.4. The first identity is due essentially to Spivey [34], who proved it in the special case $x=1$ (the general proof is not much different from the short combinatorial argument in [34]).

Proposition 5.3. The following recurrences hold for integers $m, n \geq 0$ : 
(1) $\mathcal{B}_{m+n}(x)=\sum_{j=0}^{m} \sum_{k=0}^{n} x^{m+n-j-k} j^{n-k}\left(\begin{array}{c}n \\ k\end{array}\right)\left\{\begin{array}{c}m \\ j\end{array}\right\} \mathcal{B}_{k}(x)$.

(2)

$$
\mathcal{B}_{m+n}^{\mathrm{B}}(x)=\sum_{j=0}^{m} \sum_{k=0}^{n} x^{m+n-j-k}(2 j+1)^{n-k}\left(\begin{array}{c}
n \\
k
\end{array}\right)\left\{\begin{array}{c}
m \\
j
\end{array}\right\}_{\mathrm{B}} \mathcal{B}_{k}(2 x) .
$$

Proof. We only prove (2) as the proof of (1) is similar. Let $\mathcal{Y}=\{ \pm(m+k): k \in[n]\}$ and suppose $\mathbb{A}$ has $x+1$ elements. We may construct the elements of $\Pi^{\mathrm{B}}(m+n, \mathbb{A})$ in the following manner. First choose a partition $\Gamma$ in $\Pi^{\mathrm{B}}(m)$ with $2 j+1$ blocks; there are $\left\{\begin{array}{c}m \\ j\end{array}\right\}_{\mathrm{B}}$ choices for this. Next, select a $2(n-k)$-element subset $S \subset \mathcal{Y}$ with $S=-S$ and distribute the elements of $S$ among the blocks of $\Gamma$ so that the resulting partition $\Gamma^{\prime}$ has $\Gamma^{\prime}=-\Gamma^{\prime}$; there are $\left(\begin{array}{l}n \\ k\end{array}\right)$ choices for $S$ and $(2 j+1)^{n-k}$ possible distributions, since once we have chosen the blocks to contain the positive elements of $S$ the blocks containing the negative elements are uniquely determined. Now, label the $2(m+n-j-k) \operatorname{arcs}$ of $\Gamma^{\prime}$ by nonzero elements of $\mathbb{A}$ so that $\Gamma^{\prime}$ satisfies (5.1); there are $x^{m+n-j-k}$ such labelings. Finally, choose an $\mathbb{A}$-labeled partition of $\mathcal{Y} \backslash S$ satisfying (5.1) and concatenate this with $\Gamma^{\prime}$ to form an element of $\Pi^{\mathrm{B}}(m+n, \mathbb{A})$; there are $\mathcal{B}_{k}^{\mathrm{D}}(x)$ choices for this partition. Each element of $\Pi^{\mathrm{B}}(m+n, \mathbb{A})$ arises from exactly one such construction, so summing the product $x^{m+n-j-k}(2 j+1)^{n-k}\left(\begin{array}{l}n \\ k\end{array}\right)\left\{\begin{array}{c}m \\ j\end{array}\right\}_{\mathrm{B}} \mathcal{B}_{k}(2 x)$ over all possible values of $j$ and $k$ yields $\mathcal{B}_{m+n}^{\mathrm{B}}(x)$.

Likewise, we compute $\mathcal{N}_{n}^{\mathrm{X}}(x)$ by recalling the definition in the previous section of uncross $(\Lambda)$ for a set partition $\Lambda$. Modifying this construction slightly, for $\Lambda \in \Pi^{\mathrm{B}}(n)$, we define uncross $_{\mathrm{B}}(\Lambda)$ to be the set partition of $[ \pm n]$ formed by removing zero from its block in $\operatorname{uncross}(\Lambda)$. For example,

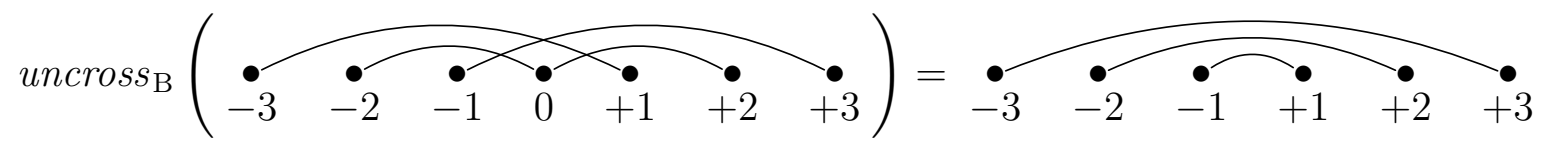

We note the following properties of these maps in the present context.

Lemma 5.4. The maps

$$
\begin{aligned}
\text { uncross }_{\mathrm{B}}: \widetilde{\mathrm{NC}}^{\mathrm{B}}(n) \rightarrow\{\text { Noncrossing set partitions } \Lambda \vdash[ \pm n] \text { with } \Lambda=-\Lambda\} \\
\text { uncross }: \widetilde{\mathrm{NC}^{\mathrm{D}}}(n) \rightarrow\left\{\begin{array}{l}
\text { Noncrossing set partitions } \Lambda \vdash[ \pm n] \text { with } \Lambda=-\Lambda \text { which } \\
\text { have an even number of blocks } B \text { such that } B=-B
\end{array}\right\}
\end{aligned}
$$

are bijections. Furthermore, if $\Lambda \in \widetilde{\mathrm{NC}}^{\mathrm{B}}(n)$ has $2 k+1$ blocks then uncross $_{\mathrm{B}}(\Lambda)$ has either $2 k$ or $2 k+1$ blocks.

Note that uncross $(\Lambda)$ has the same number of blocks as $\Lambda$ for any set partition $\Lambda$.

Proof. Given $\Lambda \vdash[ \pm n]$ with $\Lambda=-\Lambda$, let $\mathcal{X}=\left\{\left(-i_{1}, i_{1}\right), \ldots,\left(-i_{\ell}, i_{\ell}\right)\right\}$ be the set of arcs of the form $(-i, i) \in \operatorname{Arc}(\Lambda)$, where $i_{1}<\cdots<i_{\ell}$, and define $\mathcal{Y}$ as the set of arcs

$$
\mathcal{Y}= \begin{cases}\left\{\left(-i_{2 k}, i_{2 k-1}\right),\left(-i_{2 k-1}, i_{2 k}\right): k \in\left[\frac{\ell}{2}\right]\right\}, & \text { if } \ell \text { is even, } \\ \left\{\left(-i_{1}, 0\right),\left(0, i_{1}\right)\right\} \cup\left\{\left(-i_{2 k}, i_{2 k+1}\right),\left(-i_{2 k+1}, i_{2 k}\right): k \in\left[\frac{\ell-1}{2}\right]\right\}, & \text { if } \ell \text { is odd }\end{cases}
$$


Let $\Lambda^{\prime}$ be the set partition of $\{0\} \cup[ \pm n]$ with $\operatorname{arc} \operatorname{set}(\operatorname{Arc}(\Lambda)-\mathcal{X}) \cup \mathcal{Y}$, and when $\ell$ is even, let $\Lambda^{\prime \prime}$ be the set partition of $[ \pm n]$ with $\operatorname{arc} \operatorname{set}(\operatorname{Arc}(\Lambda)-\mathcal{X}) \cup \mathcal{Y}$. Then $\Lambda \mapsto \Lambda^{\prime}$ is the two-sided inverse of the first map in the lemma, while $\Lambda \mapsto \Lambda^{\prime \prime}$ is the two-sided inverse of the second map. The last remark concerning the numbers of blocks follows from the fact that uncross $_{\mathrm{B}}(\Lambda)$ partitions a set with one less element than $\Lambda$, yet has either equally many or one fewer arcs than $\Lambda$.

The first identity in the following proposition is an immediate consequence of the previous lemma and Lemma 4.1. The second part follows from the first, given the fact that $\mathcal{N}_{n+1}^{\mathrm{D}}(x)=\mathcal{N}_{n}^{\mathrm{B}}(x)+n x \mathcal{N}_{n}(x)$, an identity which we will prove in a more general form as Corollary 6.8 .

Proposition 5.5. $\mathcal{N}_{n}^{\mathrm{B}}(x)=\sum_{k=0}^{n}\left(\begin{array}{l}n \\ k\end{array}\right)^{2} x^{k}$ and $\mathcal{N}_{n+1}^{\mathrm{D}}(x)=\sum_{k=0}^{n}\left(\begin{array}{l}n \\ k\end{array}\right)\left(\begin{array}{c}n+1 \\ k\end{array}\right) x^{k}$ for $n \geq 0$.

Remark. This result shows that $\mathcal{N}_{n}^{\mathrm{B}}(x)$ is the Narayana polynomial of type $B_{n}$; i.e., the rank generating function of the lattice of noncrossing partitions of type $B_{n}$ introduced in [29]. By contrast, $\mathcal{N}_{n}^{\mathrm{D}}(x)$ is not the Narayana polynomial of type $D_{n}$; however, the latter polynomial is equal to $\mathcal{N}_{n}^{\mathrm{D}}(x)+x \mathcal{N}_{n-1}^{\mathrm{B}}(x)-x \mathcal{N}_{n-1}(x)$ for $n \geq 1$ (see [7, Theorem 1.2]). These observations imply that $\mathcal{N}_{n}^{\mathrm{B}}(1)=\left(\begin{array}{c}2 n \\ n\end{array}\right)$ and $\mathcal{N}_{n+1}^{\mathrm{D}}(1)=\left(\begin{array}{c}2 n+1 \\ n\end{array}\right)$ are central binomial coefficients.

\section{Identities in types B and D}

To apply the methods of Section 3 to our new constructions, we begin by defining the appropriate analogue of the map shift $: \Pi(n, \mathbb{A}) \rightarrow \Pi(n+1, \mathbb{A})$.

Shifting the matrix of a set partition in $\Pi^{\mathrm{B}}(n, \mathbb{A})$ (respectively, $\left.\Pi^{\mathrm{D}}(n, \mathbb{A})\right)$ one column to the right yields the matrix of a set partition in $\Pi^{\mathrm{D}}(n+1, \mathbb{A})$ (respectively, $\left.\Pi^{\mathrm{B}}(n, \mathbb{A})\right)$, and corresponds to two injective maps which, with slight abuse of notation, we again denote by shift:

$$
\text { shift }: \Pi^{\mathrm{D}}(n, \mathbb{A}) \rightarrow \Pi^{\mathrm{B}}(n, \mathbb{A}) \quad \text { and } \quad \text { shift }: \Pi^{\mathrm{B}}(n, \mathbb{A}) \rightarrow \Pi^{\mathrm{D}}(n+1, \mathbb{A}) .
$$

Explicitly:

- If $\Lambda \in \Pi^{\mathrm{D}}(n, \mathbb{A})$ then $\operatorname{shift}(\Lambda) \in \Pi^{\mathrm{B}}(n, \mathbb{A})$ has arc set $\{(f(i), f(j)+1):(i, j) \in$ $\operatorname{Arc}(\Lambda)\}$ and labeling map $(f(i), f(j)+1) \mapsto \Lambda_{i j}$, where $f(x):= \begin{cases}x, & \text { if } x<0, \\ x-1, & \text { if } x>0\end{cases}$

- If $\Lambda \in \Pi^{\mathrm{B}}(n, \mathbb{A})$ then $\operatorname{shift}(\Lambda) \in \Pi^{\mathrm{D}}(n+1, \mathbb{A})$ has arc set $\left\{\left(g(i), g(j)+\delta_{j}+1\right)\right.$ : $(i, j) \in \operatorname{Arc}(\Lambda)\}$ and labeling map $\left(g(i), g(j)+\delta_{j}+1\right) \mapsto \Lambda_{i j}$, where $g(x):=$ $\begin{cases}x-1, & \text { if } x \leq 0 \\ x, & \text { if } x>0\end{cases}$ 
One checks without difficulty that these definitions do in fact give set partitions belonging to $\Pi^{\mathrm{B}}(n, \mathbb{A})$ and $\Pi^{\mathrm{D}}(n+1, \mathbb{A})$. For example,

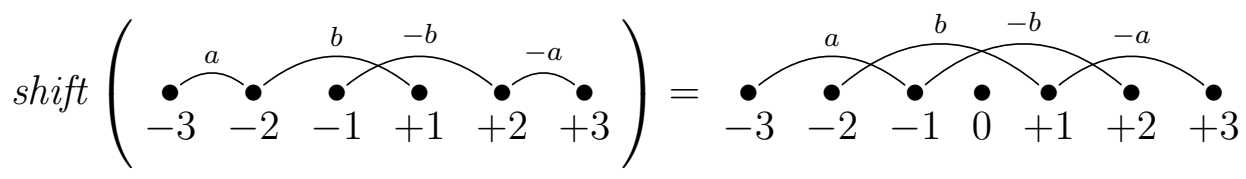

and

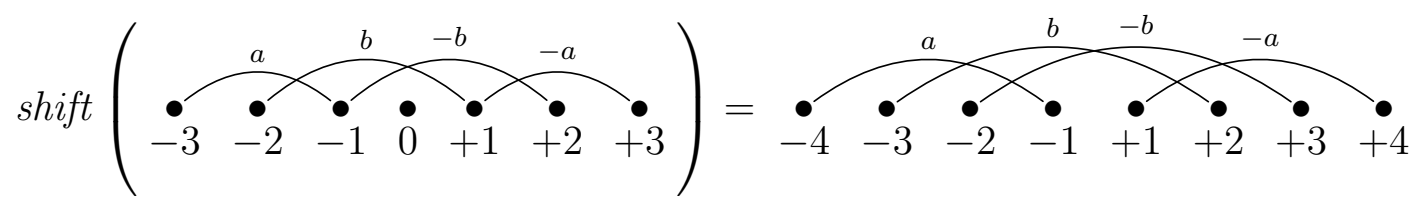

which becomes obvious after noting that the matrices of the three set partitions are

\begin{tabular}{|c|c|c|c|c|c|}
\hline 0 & $a$ & & & & \\
\hline & 0 & & $b$ & & \\
\hline & & 0 & & $-b$ & \\
\hline & & & 0 & & \\
\hline & & & & 0 & $-a$ \\
\hline & & & & & 0 \\
\hline
\end{tabular}
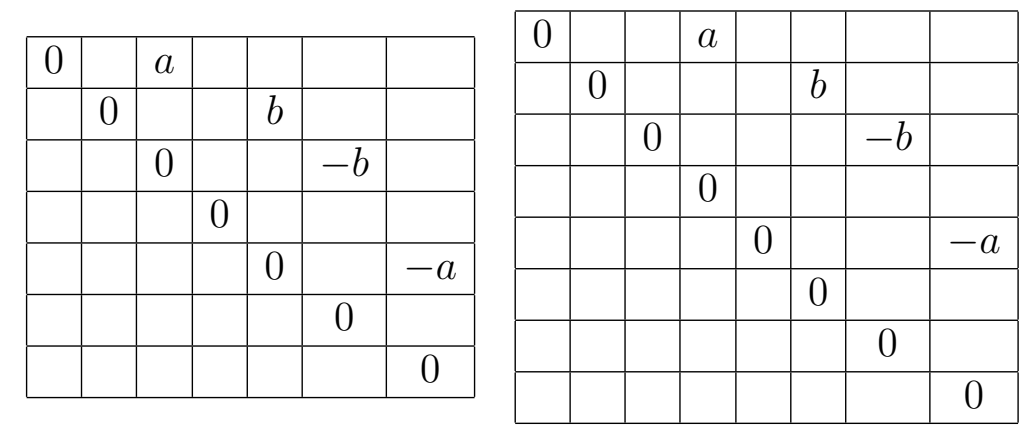

It is straightforward to see that the map shift defines bijections

$$
\begin{aligned}
& \Pi^{\mathrm{D}}(n, \mathbb{A}) \rightarrow\left\{2 \text {-regular elements of } \Pi^{\mathrm{B}}(n, \mathbb{A})\right\}, \\
& \Pi^{\mathrm{B}}(n, \mathbb{A}) \rightarrow\left\{2 \text {-regular elements of } \Pi^{\mathrm{D}}(n+1, \mathbb{A})\right\}
\end{aligned}
$$

where, as previously, a set partition is 2-regular if none of its blocks contain two consecutive integers $i$ and $i+1$. Consequently we may view shift as a map $\Pi^{\mathrm{D}}(n, \mathbb{A}) \rightarrow \Pi^{\mathrm{B}}(n, \mathbb{A}, \mathbb{B})$ and $\Pi^{\mathrm{B}}(n, \mathbb{A}) \rightarrow \Pi^{\mathrm{D}}(n+1, \mathbb{A}, \mathbb{B})$.

We define the feasible and poor elements of $\Pi^{\mathrm{B}}(n)$ or $\Pi^{\mathrm{D}}(n)$ exactly as for $\Pi(n)$. In addition, we say that a set partition is $B$-feasible if none of its blocks contain exactly one nonzero element and $B$-poor if none of its blocks contain more than two nonzero elements. Observe that these notions are distinct from "feasible" and "poor" only for elements of $\Pi^{\mathrm{B}}(n)$. The following lemma, in analogy with Lemma 3.1, describes the action of shift on these sets of interest.

Lemma 6.1. The following restrictions of shift are bijections:

(1) $\left\{\right.$ Feasible elements of $\left.\Pi^{\mathrm{D}}(n, \mathbb{A})\right\} \rightarrow\left\{\begin{array}{l}\text { 2-regular partitions } \Lambda \in \Pi^{\mathrm{B}}(n, \mathbb{A}) \text { such that } \\ 1+\max B \neq \min B^{\prime} \text { for all blocks } B, B^{\prime} \in \Lambda\end{array}\right\}$. 
(2) $\left\{\right.$ B-feasible elements of $\left.\Pi^{\mathrm{B}}(n, \mathbb{A})\right\} \rightarrow\left\{\begin{array}{l}\text { 2-regular partitions } \Lambda \in \Pi^{\mathrm{D}}(n+1, \mathbb{A}) \text { with } \\ 1+\max B \neq \min B^{\prime} \text { for all blocks } B, B^{\prime} \in \Lambda\end{array}\right\}$.

(3) $\left\{\right.$ Poor elements of $\left.\widetilde{\mathrm{NC}^{\mathrm{D}}}(n, \mathbb{A})\right\} \rightarrow\left\{\right.$ 2-regular elements of $\left.\widetilde{\mathrm{NC}}^{\mathrm{B}}(n, \mathbb{A})\right\}$.

(4) $\left\{\right.$ B-poor elements of $\left.\widetilde{\mathrm{NC}^{\mathrm{B}}}(n, \mathbb{A})\right\} \rightarrow\left\{\right.$ 2-regular elements of $\left.\widetilde{\mathrm{NC}}^{\mathrm{D}}(n+1, \mathbb{A})\right\}$.

Proof. Parts (1) and (2) follow from the intuitive definition of shift on matrices after noting that

- A partition $\Lambda$ in $\Pi^{\mathrm{D}}(n, \mathbb{A})$ (respectively, $\left.\Pi^{\mathrm{B}}(n, \mathbb{A})\right)$ is feasible (respectively, B-feasible) if and only if its matrix has a nonzero entry in either the $i$ th row or $i$ th column for each $i \in[2 n]$ (respectively, for each $i \in[2 n+1] \backslash\{n+1\}$ ).

- A partition $\Lambda$ in $\Pi^{\mathrm{B}}(n, \mathbb{A})$ (respectively, $\left.\Pi^{\mathrm{D}}(n+1, \mathbb{A})\right)$ is 2-regular and has the property that $1+\max B \neq \min B^{\prime}$ for all blocks $B, B^{\prime} \in \Lambda$ if and only if its matrix has no nonzero entries on the superdiagonal but has at least one nonzero entry in the superdiagonal hook $\{(i, j+1): i<j\} \cup\{(j, k): j<k\}$ for each $i \in[2 n]$ (respectively, for each $i \in[2 n+2] \backslash\{n+1\})$.

Parts (3) and (4) follow from similar considerations.

For $\mathrm{X} \in\{\mathrm{B}, \mathrm{D}\}$, we let $\mathcal{F}_{n}^{\mathrm{X}}(x), \widetilde{\mathcal{F}}_{n}^{\mathrm{B}}(x), \mathcal{M}_{n}^{\mathrm{X}}(x)$, and $\widetilde{\mathcal{M}}_{n}^{\mathrm{B}}(x)$ denote the polynomials

$$
\begin{array}{llrl}
\mathcal{F}_{n}^{\mathrm{X}}(x) & =\sum_{\text {Feasible } \Lambda \in \Pi^{\mathrm{X}}(n)} x^{\frac{|\operatorname{Arc}(\Lambda)|}{2}} & \mathcal{M}_{n}^{\mathrm{X}}(x) & =\sum_{\operatorname{Poor} \Lambda \in \widetilde{\mathrm{NC}^{\mathrm{X}}}(n)} x^{\frac{|\operatorname{Arc}(\Lambda)|}{2}} \\
\widetilde{\mathcal{F}}_{n}^{\mathrm{B}}(x)=\sum_{\text {B-feasible } \Lambda \in \widetilde{\mathrm{NC}^{\mathrm{B}}}(n)} x^{\frac{|\operatorname{Arc}(\Lambda)|}{2}} & \text { and } & \widetilde{\mathcal{M}}_{n}^{\mathrm{B}}(x)=\sum_{\text {B-poor } \Lambda \in \widetilde{\mathrm{NC}}^{\mathrm{B}}(n)} x^{\frac{|\operatorname{Arc}(\Lambda)|}{2}} .
\end{array}
$$

The next few results provide more explicit formulas for these functions.

To begin, observe that in analogy with $(5.2)$, we have

$$
\mathcal{F}_{n}^{\mathrm{D}}(x)=\mathcal{F}_{n}(2 x), \quad \text { for all } n \geq 0 \text {. }
$$

In a different direction, note that a poor set partition in $\Pi^{\mathrm{B}}(n)$ must contain the singleton $\{0\}$ as a block, and removing this block defines a bijection from the set of poor elements of $\Pi^{\mathrm{B}}(n)$ to the set of poor elements of $\Pi^{\mathrm{D}}(n)$. The first equality in the next proposition derives from this fact.

Proposition 6.2. $\mathcal{M}_{n}^{\mathrm{B}}(x)=\mathcal{M}_{n}^{\mathrm{D}}(x)=\sum_{k=0}^{\lfloor n / 2\rfloor}\left(\begin{array}{c}2 k \\ k\end{array}\right)\left(\begin{array}{c}n \\ 2 k\end{array}\right) x^{k}$.

Remark. The numbers $\left\{\mathcal{M}_{n}^{\mathrm{B}}(1)\right\}_{n=0}^{\infty}=(1,1,3,7,19,51,141, \ldots)$ are the central trinomial coefficients [33, A002426], defined as the coefficient of $x^{n}$ in $\left(1+x+x^{2}\right)^{n}$. 
Proof. Since a poor element of $\Pi^{\mathrm{D}}(n)$ with $2 k$ arcs has $2(n-k)$ singleton blocks which come in pairs $\{i\},\{-i\}$, we have $\mathcal{M}_{n}^{\mathrm{D}}(x)=\sum_{k}\left(\begin{array}{l}n \\ k\end{array}\right)\left|\mathcal{X}_{k}\right| x^{k}$ where $\mathcal{X}_{k}$ is the set of poor partitions in $\widetilde{\mathrm{NC}^{\mathrm{D}}}(k)$ whose blocks all have size two. By Lemma 5.4, the map uncross defines a bijection $\mathcal{X}_{k} \rightarrow \mathcal{Y}_{k}$, where $\mathcal{Y}_{k}$ is the set of noncrossing set partitions $\Lambda \vdash[ \pm k]$ with $\Lambda=-\Lambda$ which have $k$ blocks of size two and an even number of blocks of the form $\{-i, i\}$. To prove the proposition it suffices to show that $\mathcal{Y}_{k}$ is empty if $k$ is odd and that $\left|\mathcal{Y}_{2 k}\right|=\left(\begin{array}{c}2 k \\ k\end{array}\right)$.

Since the elements of $\mathcal{Y}_{k}$ are noncrossing and invariant under negation, the blocks of a partition $\Lambda \in \mathcal{Y}_{k}$ which are not of the form $\{-i, i\}$ are of the form $\{i, j\}$ with $i, j>0$ or $i, j<0$. Hence, removing all blocks of $\Lambda \in \mathcal{Y}_{k}$ which contain negative integers (and then shifting indices) produces a noncrossing partition of $[k-\ell]$ whose blocks all have size two, where $\ell$ is the number of blocks of $\Lambda$ of the form $\{-i, i\}$. Since $\ell$ is always even, if $k$ is odd then no such partitions exist and $\left|\mathcal{Y}_{k}\right|=0$.

Let $\mathcal{Z}_{k}$ be the set of all noncrossing set partitions of $[2 k]$ whose blocks all have size two. Given $\Lambda \in \mathcal{Z}_{k}$, let $\varphi(\Lambda) \in \mathcal{D}_{k}$ be the Dyck path whose $i$ th step is $(1,1)$ if $i$ is the smaller of the two elements in its part of $\Lambda$ and $(1,-1)$ otherwise. One checks that $\varphi: \mathcal{Z}_{k} \rightarrow \mathcal{D}_{k}$ is a well-defined bijection (one recovers $\Lambda$ by numbering the steps of $\varphi(\Lambda)$ from 1 to $2 k$ and placing the pairs of numbers indexing each upstep $(1,1)$ and the following downstep $(1,-1)$ at the same height in blocks), and it is clear that if we view $\mathcal{Y}_{2 k}$ as a subset of $\mathcal{Z}_{2 k}$, then $\varphi$ restricts to a bijection from $\mathcal{Y}_{2 k}$ to the set of symmetric elements of $\mathcal{D}_{2 k}$. Hence $\left|\mathcal{Y}_{2 k}\right|=\left(\begin{array}{c}2 k \\ k\end{array}\right)$ by Lemma 4.2 , as required.

Inspecting the formulas for $\mathcal{M}_{n}(x)$ and $\mathcal{M}_{n}^{\mathrm{B}}(x)$ in Propositions 3.2 and 6.2 yields the following trivial but useful corollary.

Corollary 6.3. $\mathcal{M}_{n+2}^{\mathrm{B}}(x)=\mathcal{M}_{n+1}^{\mathrm{B}}(x)+2(n+1) x \mathcal{M}_{n}(x)$ for $n \geq 0$.

We now turn our attention to the polynomials $\widetilde{\mathcal{F}}_{n}^{\mathrm{B}}(x)$ and $\widetilde{\mathcal{M}}_{n}^{\mathrm{B}}(x)$ counting B-feasible and B-poor noncrossing partitions in $\Pi^{\mathrm{B}}(n, \mathbb{A})$.

Proposition 6.4. The following identities hold for $n \geq 0$ :

(1) $\widetilde{\mathcal{F}}_{n}^{\mathrm{B}}(x)=\mathcal{F}_{n}^{\mathrm{B}}(x)+\mathcal{F}_{n}(2 x)=\sum_{k=0}^{n}\left(\begin{array}{l}n \\ k\end{array}\right) \mathcal{F}_{k}(2 x) x^{n-k}$.

(2) $\widetilde{\mathcal{M}}_{n}^{\mathrm{B}}(x)=\mathcal{M}_{n}^{\mathrm{B}}(x)+n x \mathcal{M}_{n-1}(x)=\sum_{k=0}^{\lceil n / 2\rceil}\left(\begin{array}{l}n \\ k\end{array}\right)\left(\begin{array}{c}n+1-k \\ k\end{array}\right) x^{k}$.

Remark. We have $\widetilde{\mathcal{F}}_{n}^{\mathrm{B}}(1)=\mathcal{B}_{n}(2)$ since splitting the block containing 0 into singletons and then removing $\{0\}$ defines a bijection from the set of B-feasible elements of $\Pi^{\mathrm{B}}(n)$ to $\Pi^{\mathrm{D}}(n)$; this also follows from the first remark to Theorem 3.4. The numbers $\left\{\widetilde{\mathcal{M}}_{n}^{\mathrm{B}}(1)\right\}_{n=0}^{\infty}=(1,1,2,5,13,35,96, \ldots)$ count the directed animals of size $n+1[33$, A005773]. 
Proof. A B-feasible element of $\Pi^{\mathrm{B}}(n)$ is either feasible or contains $\{0\}$ as a block, and removing the block $\{0\}$ gives a bijection from the partitions of the latter kind to the feasible elements of $\Pi^{\mathrm{D}}(n)$. We obtain the second equality in (1) by noting that $\left(\begin{array}{l}n \\ k\end{array}\right) \mathcal{F}_{k}(2 x) x^{n-k}$ is the number of B-feasible elements of $\Pi^{\mathrm{B}}(n)$ in which 0 belongs to a block with exactly $2 k+1$ elements.

For part (2), we observe that $\widetilde{\mathcal{M}}_{n}^{\mathrm{B}}(x)=\mathcal{M}_{n}^{\mathrm{B}}(x)+\sum_{k=1}^{n} \widetilde{\mathcal{M}}_{n, k}^{\mathrm{B}}(x)$ where $\widetilde{\mathcal{M}}_{n, k}^{\mathrm{B}}(x)$ is the sum of $x^{\frac{|\operatorname{Arc}(\Lambda)|}{2}}$ over all B-poor set partitions $\Lambda \in \widetilde{\mathrm{NC}^{\mathrm{B}}}(n)$ which possess $\{-k, 0, k\}$ as a block. In such partitions, all remaining blocks are subsets of either $\{1,2, \ldots, k-1\}$, $\{-1,-2, \ldots,-k+1\}$, or $[ \pm n] \backslash[ \pm k]$. The blocks contained in the first two of these sets are reflections of each other and determine a poor element of $\mathrm{NC}(k-1)$, while the blocks contained in $[ \pm n] \backslash[ \pm k]$ determine a poor element of $\widetilde{N C}^{\mathrm{D}}(n-k)$. It follows that $\widetilde{\mathcal{M}}_{n, k}^{\mathrm{B}}(x)=x \mathcal{M}_{k-1}(x) \mathcal{M}_{n-k}^{\mathrm{B}}(x)$.

By considering whether the blocks of $\pm(n+2)$ in a poor element of $\widetilde{\mathrm{NC}}^{\mathrm{D}}(n+2, \mathbb{A})$ are singletons or contain a second element in $[ \pm(n+1)]$, one obtains the recurrence $\mathcal{M}_{n+2}^{\mathrm{B}}(x)=\mathcal{M}_{n+1}^{\mathrm{B}}(x)+2 x \sum_{k=0}^{n} \mathcal{M}_{k}(x) \mathcal{M}_{n-k}^{\mathrm{B}}(x)$ for $n \geq 0$. Subtracting the expression in Corollary 6.3 from this equation shows that $\sum_{k=1}^{n} \widetilde{\mathcal{M}}_{n, k}^{\mathrm{B}}(x)=n x \mathcal{M}_{n-1}(x)$, which then gives the formula in (2).

The following corollary will be of use in the proof of Theorem 6.7 .

Corollary 6.5. The following identities hold for $n \geq 0$ :

(1) There are $\left(\begin{array}{c}2 n \\ n\end{array}\right)$ elements of $\widetilde{N C}^{\mathrm{D}}(2 n)$ whose blocks all have size two.

(2) There are no elements of $\widetilde{\mathrm{NC}}^{\mathrm{D}}(2 n+1)$ whose blocks all have size two.

(3) There are $\left(\begin{array}{c}n \\ \lfloor n / 2\rfloor\end{array}\right)$ B-poor elements of $\widetilde{N C}^{\mathrm{B}}(n)$ with no nonzero singleton blocks.

Proof. All blocks of an element of $\widetilde{\mathrm{NC}}^{\mathrm{D}}(n)$ have size two if and only if the partition has exactly $n$ arcs; hence, the number counted in part (1) is the coefficient of $x^{n / 2}$ in $\mathcal{M}_{n}^{\mathrm{D}}(x)$. An element of $\widetilde{\mathrm{NC}^{\mathrm{B}}}(n)$ is B-poor and has no nonzero singleton blocks if and only if either the partition contains $\{0\}$ as a block such that removing this block produces an element of $\widetilde{\mathrm{NC}}^{\mathrm{D}}(n)$ whose blocks all have size two, or if the partition has $n+1$ arcs. Hence the number counted in part (2) is the sum of the number in (1) and the coefficient of $x^{(n+1) / 2}$ in $\widetilde{\mathcal{M}}_{n}^{\mathrm{B}}(x)$.

Serving as an analogue for Theorem 3.3, the following result shows that $\mathcal{B}_{n}(2 x)$ and $\mathcal{M}_{n}^{\mathrm{B}}(x)$ count the $\mathrm{L}^{\mathrm{B}}(n, \mathbb{B})$-orbits in $\Pi^{\mathrm{B}}(n, \mathbb{A}, \mathbb{B})$ and $\widetilde{\mathrm{NC}^{\mathrm{B}}}(n, \mathbb{A}, \mathbb{B})$, while $\mathcal{B}_{n}^{\mathrm{B}}(x)$ and $\widetilde{\mathcal{M}}_{n}^{\mathrm{B}}(x)$ count the $\mathrm{L}^{\mathrm{D}}(n+1, \mathbb{B})$-orbits in $\Pi^{\mathrm{D}}(n+1, \mathbb{A}, \mathbb{B})$ and $\widetilde{\mathrm{NC}}^{\mathrm{D}}(n+1, \mathbb{A}, \mathbb{B})$.

Theorem 6.6. Let $n$ be a positive integer. 
(1) The correspondence $\Lambda \mapsto\left\{\alpha+\operatorname{shift}(\Lambda): \alpha \in \mathrm{L}^{\mathrm{B}}(n, \mathbb{B})\right\}$ is a bijection

$$
\begin{aligned}
& \Pi^{\mathrm{D}}(n, \mathbb{A}) \rightarrow\left\{\mathrm{L}^{\mathrm{B}}(n, \mathbb{B}) \text {-orbits in } \Pi^{\mathrm{B}}(n, \mathbb{A}, \mathbb{B})\right\}, \\
&\left\{\text { Poor elements of } \widetilde{\left.\mathrm{NC}^{\mathrm{D}}(n, \mathbb{A})\right\}} \rightarrow\left\{\mathrm{L}^{\mathrm{B}}(n, \mathbb{B}) \text {-orbits in } \widetilde{\mathrm{NC}^{\mathrm{B}}}(n, \mathbb{A}, \mathbb{B})\right\} .\right.
\end{aligned}
$$

Furthermore, the cardinality of the $\mathrm{L}^{\mathrm{B}}(n, \mathbb{B})$-orbit of $\operatorname{shift}(\Lambda)$ is $|\mathbb{B}|^{s / 2}$, where $s$ is the number of singleton blocks of $\Lambda \in \Pi^{\mathrm{D}}(n, \mathbb{A})$.

(2) The correspondence $\Lambda \mapsto\left\{\alpha+\operatorname{shift}(\Lambda): \alpha \in \mathrm{L}^{\mathrm{D}}(n, \mathbb{B})\right\}$ is a bijection

$$
\begin{aligned}
& \Pi^{\mathrm{B}}(n-1, \mathbb{A}) \rightarrow\left\{\mathrm{L}^{\mathrm{D}}(n, \mathbb{B}) \text {-orbits in } \Pi^{\mathrm{D}}(n, \mathbb{A}, \mathbb{B})\right\}, \\
& \left\{\text { B-poor elements of } \widetilde{\mathrm{NC}^{\mathrm{B}}}(n-1, \mathbb{A})\right\} \rightarrow\left\{\mathrm{L}^{\mathrm{D}}(n, \mathbb{B}) \text {-orbits in } \widetilde{\mathrm{NC}^{\mathrm{D}}}(n, \mathbb{A}, \mathbb{B})\right\} \text {. }
\end{aligned}
$$

The cardinality of the $\mathrm{L}^{\mathrm{D}}(n, \mathbb{B})$-orbit of $\operatorname{shift}(\Lambda)$ is $|\mathbb{B}|^{\lfloor s / 2\rfloor}$, where $s$ is the number of singleton blocks of $\Lambda \in \Pi^{\mathrm{B}}(n-1, \mathbb{A})$.

In part (2), $\lfloor s / 2\rfloor$ is half the number of nonzero singleton blocks of $\Lambda \in \Pi^{\mathrm{B}}(n-1, \mathbb{A})$.

Proof. The proof is quite similar to that of Theorem 3.3. Let $\mathrm{X} \in\{\mathrm{B}, \mathrm{D}\}$. As in the earlier proof, the definition of + implies that each $\mathrm{L}^{\mathrm{X}}(n, \mathbb{B})$-orbit in $\Pi^{\mathrm{X}}(n, \mathbb{A}, \mathbb{B})$ contains a unique 2-regular element, and this suffices by (6.1) and Lemma 6.1 to show that the maps in (1) and (2) are bijections.

If $\alpha \in \mathrm{L}^{\mathrm{X}}(n, \mathbb{B})$ then $(i, i+1) \in \operatorname{Arc}(\alpha)$ if and only if $(-i-1,-i) \in \operatorname{Arc}(\alpha)$ and the pair of $\operatorname{arcs}(i, i+1),(-i-1,-i)$ have opposite labels. Also, if $\alpha \in \mathrm{L}^{\mathrm{D}}(n, \mathbb{B})$ then $(-1,1)$ never belongs to $\operatorname{Arc}(\alpha)$. Consequently, from our definition of + via Observation 2.2 , it follows that if $\Lambda$ belongs to $\Pi^{\mathrm{D}}(n, \mathbb{A})$ (respectively, $\left.\Pi^{\mathrm{B}}(n-1, \mathbb{A})\right)$ then the orbit of $\operatorname{shift}(\Lambda)$ has size $|\mathbb{B}|^{s / 2}$, where $s$ is the number the superdiagonal hooks $\{(i, j+1): i<j\} \cup\{(j, k): j<k\}$ for $j \in[2 n]$ (respectively, for $j \in[2 n-1] \backslash\{n\}$ ) containing no nonzero entries in the matrix of shift $(\Lambda)$. In both cases, the intuitive definition of shift implies that $s$ is the (even) number of nonzero singleton blocks in $\Lambda$.

We may now prove the type B and D analogue of Theorem 3.4 .

Theorem 6.7. If $n$ is a nonnegative integer then the following identities hold:

(1) $\mathcal{B}_{n}^{\mathrm{B}}(x, y)=\sum_{k=0}^{n}\left(\begin{array}{l}n \\ k\end{array}\right) \mathcal{B}_{k}(2 x) y^{n-k}=\sum_{k=0}^{n}\left(\begin{array}{l}n \\ k\end{array}\right) \mathcal{F}_{k}(2 x)(y+1)^{n-k}$.

$$
\begin{aligned}
& \text { (2) } \mathcal{B}_{n+1}^{\mathrm{D}}(x, y)=\sum_{k=0}^{n}\left(\begin{array}{l}
n \\
k
\end{array}\right) \mathcal{B}_{k}^{\mathrm{B}}(x) y^{n-k}=\sum_{k=0}^{n}\left(\begin{array}{l}
n \\
k
\end{array}\right) \widetilde{\mathcal{F}}_{k}^{\mathrm{B}}(x)(y+1)^{n-k} . \\
& \text { (3) } \mathcal{N}_{n}^{\mathrm{B}}(x, y)=\sum_{k=0}^{n}\left(\begin{array}{l}
n \\
k
\end{array}\right) \mathcal{M}_{k}^{\mathrm{B}}(x) y^{n-k}=\sum_{k=0}^{n}\left(\begin{array}{c}
2 k \\
k
\end{array}\right)\left(\begin{array}{c}
n \\
2 k
\end{array}\right) x^{k}(y+1)^{n-2 k} .
\end{aligned}
$$


(4) $\mathcal{N}_{n+1}^{\mathrm{D}}(x, y)=\sum_{k=0}^{n}\left(\begin{array}{l}n \\ k\end{array}\right) \widetilde{\mathcal{M}}_{k}^{\mathrm{B}}(x) y^{n-k}=\sum_{k=0}^{n}\left(\begin{array}{l}n \\ k\end{array}\right)\left(\begin{array}{c}k \\ \lfloor k / 2\rfloor\end{array}\right) x^{\lceil k / 2\rceil}(y+1)^{n-k}$.

Proof. As in the proof of Theorem 3.4, if $|\mathbb{A}|=x+1$ and $|\mathbb{B}|=y+1$, then in each part, the terms in first sum counts partitions (in $\Pi^{\mathrm{B}}(n, \mathbb{A}, \mathbb{B}), \Pi^{\mathrm{D}}(n+1, \mathbb{A}, \mathbb{B}), \widetilde{\mathrm{NC}^{\mathrm{B}}}(n, \mathbb{A}, \mathbb{B})$, or $\widetilde{N C}^{\mathrm{D}}(n+1, \mathbb{A}, \mathbb{B})$, respectively) whose matrices have the same number of nonzero entries on the superdiagonal, while the terms in the second sum count partitions whose $\mathrm{L}^{\mathrm{B}}(n, \mathbb{B})$ - or $\mathrm{L}^{\mathrm{D}}(n+1, \mathbb{B})$-orbits have the same fixed size. Checking the details of this assertion-using Corollary 6.5, Equation 6.1), Lemma 6.1, and Theorem 6.6-is entirely analogous to the proof of Theorem 3.4, and we leave this exercise to the reader. The sums in each part are thus necessarily equal to each other and to $\mathcal{B}_{n}^{\mathrm{B}}(x, y)$ in $(1), \mathcal{B}_{n+1}^{\mathrm{D}}(x, y)$ in $(2), \mathcal{N}_{n}^{\mathrm{B}}(x, y)$ in $(3)$, and $\mathcal{N}_{n+1}^{\mathrm{D}}(x, y)$ in $(4)$.

Remark. We may rewrite part (3) as the following identity, first proved in a different way by Chen, Wang, and Zhao [15, Theorem 2.5]:

$$
\sum_{i=0}^{n} \sum_{j=0}^{\left\lfloor\frac{n-i}{2}\right\rfloor}\left(\begin{array}{c}
n \\
i
\end{array}\right)\left(\begin{array}{c}
n-i \\
j
\end{array}\right)\left(\begin{array}{c}
n-i-j \\
j
\end{array}\right) x^{j} y^{i}=\sum_{k=0}^{\lfloor n / 2\rfloor}\left(\begin{array}{c}
2 k \\
k
\end{array}\right)\left(\begin{array}{c}
n \\
2 k
\end{array}\right) x^{k}(y+1)^{n-2 k} .
$$

Setting $x=y$ here recovers the identity 1.3 mentioned in the introduction, and taking $x=y=1$ gives $\left(\begin{array}{c}2 n \\ n\end{array}\right)=\sum_{k}\left(\begin{array}{c}n \\ 2 k\end{array}\right)\left(\begin{array}{c}2 k \\ k\end{array}\right) 2^{n-2 k}$, a type B analogue for Touchard's formula for the Catalan numbers noted by Simion [32].

Substituting the expressions for $\mathcal{M}_{k}(x)$ and $\widetilde{\mathcal{M}}_{k}^{\mathrm{B}}(x)$ given in Corollary 6.3 and Proposition 6.4 into parts (3) and (4) of the preceding result, while noting the second part of Theorem 3.4, leads to the next corollary.

Corollary 6.8. The following identities hold for $n \geq 0$ :

(1) $\mathcal{N}_{n+1}^{\mathrm{B}}(x, y)=(y+1) \mathcal{N}_{n}^{\mathrm{B}}(x, y)+2 n x \mathcal{N}_{n}(x, y)$.

(2) $\mathcal{N}_{n+1}^{\mathrm{D}}(x, y)=\mathcal{N}_{n}^{\mathrm{B}}(x, y)+n x \mathcal{N}_{n}(x, y)$.

This brings to light some redundancy in our identities. The first part provides a way to derive (1.2) from (1.3) in the introduction, and the second part shows that the equality of the two expressions in part (4) of Theorem 6.7 follows by taking an appropriate linear combination of part (2) of Theorem 3.4 and part (3) of Theorem 6.7.

As a second corollary, we have this analogue of Corollary 3.5 .

Corollary 6.9. If $n$ is a nonnegative integer then the following identities hold:

(1) $\sum_{k=0}^{n}(-1)^{n-k}\left(\begin{array}{l}n \\ k\end{array}\right)(y+1)^{n-k} \mathcal{B}_{k}^{\mathrm{B}}(x, y)=\mathcal{F}_{n}(2 x)$.
(2) $\sum_{k=0}^{n}(-1)^{n-k}\left(\begin{array}{l}n \\ k\end{array}\right)(y+1)^{n-k} \mathcal{B}_{k+1}^{\mathrm{D}}(x, y)=\widetilde{\mathcal{F}}_{n}^{\mathrm{B}}(x)$. 
(3) $\sum_{k=0}^{n}(-1)^{n-k}\left(\begin{array}{l}n \\ k\end{array}\right)(y+1)^{n-k} \mathcal{N}_{k}^{\mathrm{B}}(x, y)= \begin{cases}\left(\begin{array}{c}n \\ n / 2\end{array}\right) x^{n / 2}, & \text { if } n \text { is even } \\ 0, & \text { if } n \text { is odd }\end{cases}$

(4) $\sum_{k=0}^{n}(-1)^{n-k}\left(\begin{array}{l}n \\ k\end{array}\right)(y+1)^{n-k} \mathcal{N}_{k+1}^{\mathrm{D}}(x, y)=\left(\begin{array}{c}n \\ \lfloor n / 2\rfloor\end{array}\right) x^{\lceil n / 2\rceil}$.

Remark. Setting $x=y$ in part (3) produces the result given by Chen, Wang, and Zhao as [15, Theorem 3.1]. Setting $x=y=1$ in this equation gives an identity attributed to Dawson [30, Page 71], for which Andrews [4, Theorem 5.4] gives a proof using basic hypergeometric functions.

Proof. The proof is almost the same as that of Corollary 3.5. In short, letting given a subset $S \subset[n]$, let $\mathcal{X}_{S}$ and $\mathcal{Y}_{S}$ denote the unions of the $\mathrm{L}^{\mathrm{B}}(n, \mathbb{B})$-orbits of shift $(\Lambda)$ for all partitions $\Lambda$ in $\Pi^{\mathrm{D}}(n, \mathbb{A})$ and $\widetilde{\mathrm{NC}}^{\mathrm{D}}(n, \mathbb{A})$, respectively, which contain the singletons $\{i\}$ and $\{-i\}$ as blocks for each $i \in S$. By Theorem 6.6 the sets of $\mathrm{L}^{\mathrm{B}}(n, \mathbb{B})$-invariants in $\Pi^{\mathrm{B}}(n, \mathbb{A}, \mathbb{B})$ and $\widetilde{\mathrm{NC}^{\mathrm{B}}}(n, \mathbb{A}, \mathbb{B})$ are the respective complements of $\bigcup_{i \in[n]} \mathcal{X}_{\{i\}}$ and $\bigcup_{i \in[n]} \mathcal{Y}_{\{i\}}$. Using Theorems 6.6 and 6.7 and the inclusion-exclusion principle to count the elements in these unions affords parts (1) and (3) upon setting $x=|\mathbb{A}|-1$ and $y=|\mathbb{B}|-1$.

One proves parts (2) and (4) in the same way, by considering the analogous sets $\mathcal{X}_{S}^{\prime}$ and $\mathcal{Y}_{S}^{\prime}$ given by the unions of the $\mathrm{L}^{\mathrm{D}}(n+1, \mathbb{B})$-orbits of $\operatorname{shift}(\Lambda)$ for all partitions $\Lambda$ in $\Pi^{\mathrm{B}}(n, \mathbb{A})$ and $\widetilde{\mathrm{NC}^{\mathrm{B}}}(n, \mathbb{A})$, respectively, which contain the singletons $\{i\}$ and $\{-i\}$ as blocks for each $i \in S$.

We conclude this section by noting the following recurrences for $\mathcal{N}_{n}(x, y)$ and $\mathcal{N}_{n}^{\mathrm{B}}(x, y)$, which are easy consequences of the preceding results but which are difficult to deduce otherwise.

Corollary 6.10. For $n \geq 2$, the following recurrences hold:

(1) $(n+1) \mathcal{N}_{n}(x, y)=(y+1)(2 n-1) \mathcal{N}_{n-1}(x, y)+\left(4 x-(y+1)^{2}\right)(n-2) \mathcal{N}_{n-2}(x, y)$.

(2) $n \mathcal{N}_{n}^{\mathrm{B}}(x, y)=(y+1)(2 n-1) \mathcal{N}_{n-1}^{\mathrm{B}}(x, y)+\left(4 x-(y+1)^{2}\right)(n-1) \mathcal{N}_{n-2}^{\mathrm{B}}(x, y)$.

Remark. Sulanke provides bijective proofs of part (1) in the increasingly general cases $x=y \in\{1,2\}$ [36]; $x=1$ [37, Proposition 1.1]; and $x=y$ [38].

Proof. After substituting the right-most expressions in Theorem 3.4 and Theorem 6.7 for $\mathcal{N}_{n}(x, y)$ and $\mathcal{N}_{n}^{\mathrm{B}}(x, y)$ into these equations, the theorem is equivalent to easily checked identities involving binomial coefficients and Catalan numbers.

\section{Connections to representation theory}

Fix a finite field $\mathbb{F}_{q}$ with with order $q$ and odd characteristic $p>0$. Given positive integers $m, n$, we write $\mathbb{F}_{q}^{m \times n}$ for the set of $m \times n$ matrices over $\mathbb{F}_{q}$. For any matrix $X \in \mathbb{F}_{q}^{m \times n}$, let $X^{\dagger} \in \mathbb{F}_{q}^{n \times m}$ denote the backwards transposed matrix defined by $\left(X^{\dagger}\right)_{i j}=X_{n+1-j, m+1-i}$. 
For each positive integer $n$, let $\mathbf{U}_{n}\left(\mathbb{F}_{q}\right)$ denote the group of $n \times n$ upper triangular matrices over $\mathbb{F}_{q}$ whose diagonal entries are all equal to one, and let $\mathbf{U}_{n}^{\mathrm{B}}\left(\mathbb{F}_{q}\right) \subset \mathbf{U}_{2 n+1}\left(\mathbb{F}_{q}\right)$ and $\mathbf{U}_{n}^{\mathrm{D}}\left(\mathbb{F}_{q}\right) \subset \mathbf{U}_{2 n}\left(\mathbb{F}_{q}\right)$ be the subgroups of elements fixed by the involution $g \mapsto\left(g^{-1}\right)^{\dagger}$. Explicitly, this means:

$$
\begin{aligned}
& \mathbf{U}_{n}\left(\mathbb{F}_{q}\right)=\left\{x \in \mathbb{F}_{q}^{n \times n}: x_{i i}=1 \text { and } x_{j i}=0 \text { for } i, j \in[n] \text { with } i<j\right\}, \\
& \mathbf{U}_{n}^{\mathrm{B}}\left(\mathbb{F}_{q}\right)=\left\{\left(\begin{array}{lll}
x & x u & x z \\
0 & 1 & -u^{\dagger} \\
0 & 0 & \left(x^{-1}\right)^{\dagger}
\end{array}\right): x \in \mathbf{U}_{n}\left(\mathbb{F}_{q}\right), u \in \mathbb{F}_{q}^{n \times 1}, z \in \mathbb{F}_{q}^{n \times n}, z^{\dagger}+z+u u^{\dagger}=0\right\}, \\
& \mathbf{U}_{n}^{\mathrm{D}}\left(\mathbb{F}_{q}\right)=\left\{\left(\begin{array}{ll}
x & x z \\
0 & \left(x^{-1}\right)^{\dagger}
\end{array}\right): x \in \mathbf{U}_{n}\left(\mathbb{F}_{q}\right), z \in \mathbb{F}_{q}^{n \times n}, z^{\dagger}+z=0\right\} .
\end{aligned}
$$

The groups $\mathbf{U}_{n+1}\left(\mathbb{F}_{q}\right), \mathbf{U}_{n}^{\mathrm{B}}\left(\mathbb{F}_{q}\right)$, and $\mathbf{U}_{n}^{\mathrm{D}}\left(\mathbb{F}_{q}\right)$ are isomorphic to the Sylow $p$-subgroups of the Chevalley groups $A_{n}(q), B_{n}(q)$, and $D_{n}(q)$.

Finding a general classification of the irreducible representations of these and related groups for all $n$ and $q$ is a well-known wild problem. However, in the past two decades a series of researchers led by C. A. M. André have defined and studied useful supercharacter theories for these groups, with many notable combinatorial properties. Introduced by Diaconis and Isaacs [20], a supercharacter theory of a finite group $G$ is a set $\mathcal{S}$ of complex characters of $G$, called supercharacters, such that (i) every irreducible character of $G$ is a constituent of exactly one character $\chi \in \mathcal{S}$; and (ii) $\mathcal{S}$ has the same cardinality as $\mathcal{K}$, the coarsest partition of $G$ with the property that each character $\chi \in \mathcal{S}$ is constant on each set $K \in \mathcal{K}$. This definition leads naturally to the idea of a "supercharacter table" and other analogues of notions from character theory.

While the groups $\mathbf{U}_{n}\left(\mathbb{F}_{q}\right), \mathbf{U}^{\mathrm{B}}\left(\mathbb{F}_{q}\right)$, and $\mathbf{U}^{\mathrm{D}}\left(\mathbb{F}_{q}\right)$ in fact have many different supercharacter theories, each has one in particular whose supercharacters are naturally indexed by $\mathbb{F}_{q}$-labeled set partitions of an appropriate type. We endeavor here to briefly describe these supercharacter theories, with the goal of lending representation theoretic meanings to some of the objects examined in the previous sections.

For a reference to the following material, see [39]. We may define the supercharacters of $\mathbf{U}_{n}\left(\mathbb{F}_{q}\right)$ by an explicit formula. Fix a nontrivial homomorphism $\theta: \mathbb{F}_{q} \rightarrow \mathbb{C}^{\times}$from the additive group of the field to the complex numbers. There is an equivalence relation on $\mathbf{U}_{n}\left(\mathbb{F}_{q}\right)$ defined by setting $x \sim y$ if and only if there are $g, h \in \mathbf{U}_{n}\left(\mathbb{F}_{q}\right)$ with $g(x-1) h=$ $y-1$. The matrices

$$
x_{\Gamma}:=1+\sum_{(i, j) \in \operatorname{Arc}(\Lambda)} \Lambda_{i j} e_{i j} \in \mathbf{U}_{n}\left(\mathbb{F}_{q}\right), \quad \text { for } \Gamma \in \Pi\left(n, \mathbb{F}_{q}\right),
$$

with $e_{i j}$ denoting an $n \times n$ elementary matrix, are representatives of the equivalence classes of this relation, which we call superclasses. For each $\Lambda \in \Pi\left(n, \mathbb{F}_{q}\right)$, let $\chi_{\Lambda}: \mathbf{U}_{n}\left(\mathbb{F}_{q}\right) \rightarrow \mathbb{C}$ 
be the function which is constant on superclasses with the formula

$$
\chi_{\Lambda}\left(x_{\Gamma}\right)= \begin{cases}\prod_{(i, l) \in \operatorname{Arc}(\Lambda)} q^{c(i, l ; \Gamma)} \theta\left(\Lambda_{i j} \Gamma_{i j}\right), & \text { if }\{(i, j),(j, k)\} \cap \operatorname{Arc}(\Gamma)=\varnothing \text { for all } i, j, k \\ 0, & \text { with } i<j<k \text { and }(i, k) \in \operatorname{Arc}(\Lambda), \\ \text { otherwise, }\end{cases}
$$

for $\Gamma \in \Pi\left(n, \mathbb{F}_{q}\right)$, where $c(i, l ; \Gamma):=l-i-1-|\{(j, k) \in \operatorname{Arc}(\Gamma): i<j<k<l\}|$ and where $\Gamma_{i j}$ is defined to be zero if $(i, j) \notin \operatorname{Arc}(\Gamma)$.

In the sequence of papers [1, 2, 3], André and Neto introduce an analogous set of supercharacters for the groups $\mathbf{U}_{n}^{\mathrm{B}}\left(\mathbb{F}_{q}\right)$ and $\mathbf{U}_{n}^{\mathrm{D}}\left(\mathbb{F}_{q}\right)$. One may succinctly define these as restrictions of certain $\chi_{\Lambda}$ 's. To state this clearly, let halve $(\Lambda)$ for $\Lambda \in \Pi^{\mathrm{B}}\left(n, \mathbb{F}_{q}\right)$ be the labeled set partition in $\Pi\left(2 n+1, \mathbb{F}_{q}\right)$ given by removing all arcs $(i, j)$ with $i+j>0$ from $\Lambda$ and then applying the natural embedding of $\mathbb{F}_{q}$-labeled partitions of $[ \pm n] \cup\{0\}$ in $\Pi\left(2 n+1, \mathbb{F}_{q}\right)$. We define halve $(\Gamma) \in \Pi\left(2 n, \mathbb{F}_{q}\right)$ for $\Gamma \in \Pi^{\mathrm{D}}\left(n, \mathbb{F}_{q}\right)$ by the same operation; i.e., remove all arcs $(i, j)$ with $i+j>0$ from $\Lambda$ and then apply the natural embedding of $\mathbb{F}_{q}$-labeled partitions of $[ \pm n]$ in $\Pi\left(2 n, \mathbb{F}_{q}\right)$. For example,

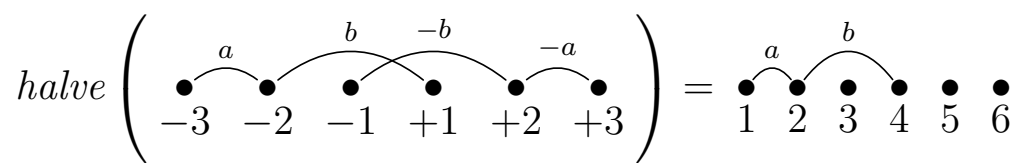

for $a, b \in \mathbb{F}_{q}^{\times}$. Now, for $\Lambda \in \Pi^{\mathrm{B}}\left(n, \mathbb{F}_{q}\right)$ and $\Gamma \in \Pi^{\mathrm{D}}\left(n, \mathbb{F}_{q}\right)$ we let $\chi_{\Lambda}^{\mathrm{B}}: \mathbf{U}_{n}^{\mathrm{B}}\left(\mathbb{F}_{q}\right) \rightarrow \mathbb{C}$ and $\chi_{\Gamma}^{\mathrm{D}}: \mathbf{U}_{n}^{\mathrm{D}}\left(\mathbb{F}_{q}\right) \rightarrow \mathbb{C}$ denote the restricted functions

$$
\chi_{\Lambda}^{\mathrm{B}}:=\operatorname{Res}_{\mathbf{U}_{n}^{\mathrm{B}}\left(\mathbb{F}_{q}\right)}^{\mathbf{U}_{2 n+1}\left(\mathbb{F}_{q}\right)}\left(\chi_{\text {halve }(\Lambda)}\right) \quad \text { and } \quad \chi_{\Gamma}^{\mathrm{D}}:=\operatorname{Res}_{\mathbf{U}_{n}^{\mathrm{D}}\left(\mathbb{F}_{q}\right)}^{\mathbf{U}_{2 n}\left(\mathbb{F}_{1}\right)}\left(\chi_{\text {halve }(\Gamma)}\right) .
$$

The significance of these functions derives from the following theorem.

Theorem 7.1. When $q$ is odd, the sets of functions

$$
\left\{\chi_{\Lambda}: \Lambda \in \Pi\left(n, \mathbb{F}_{q}\right)\right\}, \quad\left\{\chi_{\Lambda}^{\mathrm{B}}: \Lambda \in \Pi^{\mathrm{B}}\left(n, \mathbb{F}_{q}\right)\right\}, \quad \text { and } \quad\left\{\chi_{\Lambda}^{\mathrm{D}}: \Lambda \in \Pi^{\mathrm{D}}\left(n, \mathbb{F}_{q}\right)\right\}
$$

are supercharacter theories of the groups $\mathbf{U}_{n}\left(\mathbb{F}_{q}\right), \mathbf{U}_{n}^{\mathrm{B}}\left(\mathbb{F}_{q}\right)$, and $\mathbf{U}_{n}^{\mathrm{D}}\left(\mathbb{F}_{q}\right)$. These theories have $\mathcal{B}_{n}(q-1), \mathcal{B}_{n}^{\mathrm{B}}(q-1)$, and $\mathcal{B}_{n}(2 q-2)$ distinct supercharacters, respectively.

In particular, this means the functions $\chi_{\Lambda}, \chi_{\Lambda}^{\mathrm{B}}$, and $\chi_{\Lambda}^{\mathrm{D}}$ are characters. When $q$ is even, $\left\{\chi_{\Lambda}\right\}$ is still a supercharacter theory of $\mathbf{U}_{n}\left(\mathbb{F}_{q}\right)$. The methods used to show that the second two sets are supercharacter theories, however, depend on odd characteristic.

Proof. That $\left\{\chi_{\Lambda}: \Lambda \in \Pi\left(n, \mathbb{F}_{q}\right)\right\}$ is a supercharacter theory of $\mathbf{U}_{n}\left(\mathbb{F}_{q}\right)$ is well-known; see [39]. Our definition of the supercharacters $\left\{\chi_{\Lambda}^{\mathrm{B}}: \Lambda \in \Pi^{\mathrm{B}}\left(n, \mathbb{F}_{q}\right)\right\}$ and $\left\{\chi_{\Lambda}^{\mathrm{D}}: \Lambda \in\right.$ $\left.\Pi^{\mathrm{D}}\left(n, \mathbb{F}_{q}\right)\right\}$ differs from the one given by André and Neto in [1, 2, 3], but is equivalent by [2, Proposition 2.2]. Hence it follows from the main conclusions of [1, 2, 3] that both sets are supercharacter theories. 
The formula for $\chi_{\Lambda}$ shows that $\mathrm{L}\left(n, \mathbb{F}_{q}\right), \mathrm{L}^{\mathrm{B}}\left(n, \mathbb{F}_{q}\right)$, and $\mathrm{L}^{\mathrm{D}}\left(n, \mathbb{F}_{q}\right)$ index the linear supercharacters (i.e., those with degree 1 ) of $\mathbf{U}_{n}\left(\mathbb{F}_{q}\right), \mathbf{U}^{\mathrm{B}}\left(n, \mathbb{F}_{q}\right)$, and $\mathbf{U}^{\mathrm{D}}\left(n, \mathbb{F}_{q}\right)$, respectively. These supercharacters exhaust the set of all linear characters for $\mathbf{U}_{n}\left(\mathbb{F}_{q}\right)$ and $\mathbf{U}_{n}^{\mathrm{B}}\left(\mathbb{F}_{q}\right)$, but not for $\mathbf{U}_{n}^{\mathrm{D}}\left(\mathbb{F}_{q}\right)$. In addition, it is not difficult to check that the product of a supercharacter with a linear supercharacter remains a supercharacter, and that, as mentioned in the introduction, we have

$$
\chi_{\alpha} \chi_{\Lambda}=\chi_{\alpha+\Lambda}, \quad \chi_{\alpha^{\prime}}^{\mathrm{B}} \chi_{\Lambda^{\prime}}^{\mathrm{B}}=\chi_{\alpha^{\prime}+\Lambda^{\prime}}^{\mathrm{B}}, \quad \text { and } \quad \chi_{\alpha^{\prime \prime}}^{\mathrm{D}} \chi_{\Lambda^{\prime \prime}}^{\mathrm{D}}=\chi_{\alpha^{\prime \prime}+\Lambda^{\prime \prime}}^{\mathrm{D}}
$$

for $\alpha \in \mathrm{L}\left(n, \mathbb{F}_{q}\right), \alpha^{\prime} \in \mathrm{L}^{\mathrm{B}}\left(n, \mathbb{F}_{q}\right), \alpha^{\prime \prime} \in \mathrm{L}^{\mathrm{D}}\left(n, \mathbb{F}_{q}\right)$ and $\Lambda \in \Pi\left(n, \mathbb{F}_{q}\right), \Lambda^{\prime} \in \Pi^{\mathrm{B}}\left(n, \mathbb{F}_{q}\right)$, $\Lambda^{\prime \prime} \in \Pi^{\mathrm{D}}\left(n, \mathbb{F}_{q}\right)$. As a consequence, we may state the following.

Corollary 7.2. The supercharacter theories of $\mathbf{U}_{n}\left(\mathbb{F}_{q}\right), \mathbf{U}_{n}^{\mathrm{B}}\left(\mathbb{F}_{q}\right)$, and $\mathbf{U}_{n}^{\mathrm{D}}\left(\mathbb{F}_{q}\right)$ in Theorem 7.1 have $\mathcal{F}_{n-1}(q-1), \mathcal{F}_{n}(2 q-2)$, and $\widetilde{\mathcal{F}}_{n-1}^{\mathrm{B}}(q-1)$ supercharacters, respectively, which are invariant under multiplication by all linear supercharacters.

We conclude with a result which gives a representation theoretic interpretation of the sets $\widetilde{\mathrm{NC}^{\mathrm{B}}}(n, \mathbb{A})$ and $\widetilde{\mathrm{NC}^{\mathrm{D}}}(n, \mathbb{A})$ introduced in Section 5 .

Theorem 7.3. With respect to the supercharacter theories in Theorem 7.1, the following properties hold:

(1) The map $\Lambda \mapsto \chi_{\Lambda}$ defines a bijection from $\operatorname{NC}\left(n, \mathbb{F}_{q}\right)$ to the set of irreducible supercharacters of $\mathbf{U}_{n}\left(\mathbb{F}_{q}\right)$. More generally, the groups $\mathbf{U}_{n}\left(\mathbb{F}_{q}\right), \mathbf{U}_{n-1}^{\mathrm{B}}\left(\mathbb{F}_{q}\right), \mathbf{U}_{n}^{\mathrm{D}}\left(\mathbb{F}_{q}\right)$ all have exactly $\mathcal{N}_{n}(q-1)$ distinct irreducible supercharacters.

(2) The map $\Lambda \mapsto \chi_{\Lambda}^{\mathrm{B}}$ defines a bijection from $\widetilde{\mathrm{NC}}^{\mathrm{B}}\left(n, \mathbb{F}_{q}\right)$ to the set of supercharacters of $\mathbf{U}_{n}^{\mathrm{B}}\left(\mathbb{F}_{q}\right)$ not equal to the restriction of any reducible supercharacter of $\mathbf{U}_{2 n+1}\left(\mathbb{F}_{q}\right)$.

(3) The map $\Lambda \mapsto \chi_{\Lambda}^{\mathrm{D}}$ defines a bijection from $\widetilde{\mathrm{NC}}^{\mathrm{D}}\left(n, \mathbb{F}_{q}\right)$ to the set of supercharacters of $\mathbf{U}_{n}^{\mathrm{D}}\left(\mathbb{F}_{q}\right)$ not equal to the restriction of any reducible supercharacter of $\mathbf{U}_{2 n}\left(\mathbb{F}_{q}\right)$.

Proof. The first sentence in part (1) is noted in several places; e.g., 39. To show that $\mathcal{N}_{n}(q-1)$ also counts the irreducible supercharacters of $\mathbf{U}_{n+1}^{\mathrm{B}}\left(\mathbb{F}_{q}\right)$ and $\mathbf{U}_{n}^{\mathrm{D}}\left(\mathbb{F}_{q}\right)$, it suffices to show that $\chi_{\Lambda}^{\mathrm{B}}$ is irreducible if and only if $\Lambda \in \Pi^{\mathrm{B}}\left(n, \mathbb{F}_{q}\right)$ is noncrossing and $\chi_{\Lambda}^{\mathrm{D}}$ is irreducible if and only if $\Lambda \in \Pi^{\mathrm{D}}\left(n, \mathbb{F}_{q}\right)$ is noncrossing. We only address the type $\mathrm{B}$ case as the proof in type $\mathrm{D}$ is similar.

Given integers $-n \leq i<j \leq n$ with $i \neq-j$ and $t \in \mathbb{F}_{q}^{\times}$, let $\chi_{i, j, t}^{\mathrm{B}}$ denote the supercharacter of $\mathbf{U}^{\mathrm{B}}\left(n, \mathbb{F}_{q}\right)$ indexed by the labeled set partition in $\Pi^{\mathrm{B}}\left(n, \mathbb{F}_{q}\right)$ with just two $\operatorname{arcs}(i, j),(-j,-i)$ labeled by $t,-t$. From the original construction in [3, Section 2], it follows that $\chi_{\Lambda}^{\mathrm{B}}=\prod_{i, j, t} \chi_{i, j, t}^{\mathrm{B}}$, where $(i, j)$ ranges over all $(i, j) \in \operatorname{Arc}(\Lambda)$ with $i+j \leq 0$ and $t \in \mathbb{F}_{q}^{\times}$is the associated label. Moreover, if $0 \leq j<i \leq n$ and

$$
H=\left\{g \in \mathbf{U}^{\mathrm{B}}\left(n, \mathbb{F}_{q}\right): \begin{array}{l}
g_{n+1-i, k}=0 \text { if } n+1-i<k \leq n, \\
g_{n+1-j, l}=0 \text { if } n+1-j<l \leq n+1
\end{array}\right\}
$$


then, as defined in [3], $\chi_{-i, j, t}^{\mathrm{B}}$ is the character induced from the linear character $\tau$ of $H$ defined by $\tau: g \mapsto \theta\left(t g_{n+1-i, n+1+j}\right)$. In this situation, one checks that the formula for $\tau$ also defines a linear character of the group

$$
K=\left\{g \in \mathbf{U}^{\mathrm{B}}\left(n, \mathbb{F}_{q}\right): \begin{array}{l}
g_{n+1-i, k}=0 \text { if } n+1-i<k \leq n \text { and } k \neq n+1-j, \\
g_{n+1-j, l}=0 \text { if } n+1-j<l \leq n+1
\end{array}\right\} \supsetneq H
$$

whence it follows by Frobenius reciprocity and Mackey's theorem that $\chi_{-i, j, t}^{\mathrm{B}}$ has a proper constituent (given by inducing $\tau$ from $K$ ). This discussion shows that if $\Lambda \in \Pi^{\mathrm{B}}\left(n, \mathbb{F}_{q}\right)$ has an arc of the form $(-i, j)$ where $i, j>0$, then $\chi_{\Lambda}^{\mathrm{B}}$ is reducible.

If halve $(\Lambda)$ has any crossing arcs then $\chi_{\Lambda}^{\mathrm{B}}$ is reducible since it is the restriction of a reducible character. It is straightforward to see that if $\Lambda \in \Pi^{\mathrm{B}}\left(n, \mathbb{F}_{q}\right)$ has no $\operatorname{arcs}(-i, j)$ with $i, j>0$, then halve $(\Lambda)$ is noncrossing if and only if $\Lambda$ is noncrossing. In view of the previous paragraph, it follows that $\chi_{\Lambda}^{\mathrm{B}}$ is irreducible only if $\Lambda \in \Pi^{\mathrm{B}}\left(n, \mathbb{F}_{q}\right)$ is noncrossing. If $\Lambda$ is noncrossing then $\{n+i+1\}$ is a singleton block of halve $(\Lambda)$ for each $i \in[n]$. In this case, define $\Lambda^{\prime} \in \Pi\left(n+1, \mathbb{F}_{q}\right)$ as the labeled set partition formed from halve $(\Lambda)$ by removing the blocks $\{n+i+1\}$, and let $N$ be the normal subgroup of matrices $g \in \mathbf{U}_{n}^{\mathrm{B}}\left(\mathbb{F}_{q}\right)$ such that $g-1$ has all zeros in the first $n+1$ columns and in the last $n+1$ rows. The formula for $\chi_{\text {halve }(\Lambda)}$ then shows that $\chi_{\Lambda}^{\mathrm{B}}$ is obtained by inflating the supercharacter $\chi_{\Lambda^{\prime}}$ of the quotient $\mathbf{U}_{n+1}\left(\mathbb{F}_{q}\right) \cong \mathbf{U}^{\mathrm{B}}\left(n, \mathbb{F}_{q}\right) / N$. Since $\Lambda$ is noncrossing, $\Lambda^{\prime}$ is noncrossing, so both $\chi_{\Lambda^{\prime}}$ and $\chi_{\Lambda}^{\mathrm{B}}$ are irreducible. It follows that $\chi_{\Lambda}^{\mathrm{B}}$ is irreducible if and only if $\Lambda \in \Pi^{\mathrm{B}}\left(n, \mathbb{F}_{q}\right)$ is noncrossing, as required.

To prove (b), say that two partitions $\Lambda, \Lambda^{\prime} \in \Pi\left(2 n+1, \mathbb{F}_{q}\right)$ are equivalent if $\Lambda^{\prime}$ can be obtained from $\Lambda$ by replacing an $\operatorname{arc}(i, j) \in \operatorname{Arc}(\Lambda)$ labeled by $t$ with the arc $(2 n+2-$ $j, 2 n+2-i)$ labeled by $-t$; here, the arc $(i, j)$ can be replaced only if $(2 n+2-j, 2 n+2-i)$ does not already belong to $\operatorname{Arc}(\Lambda)$. If we extend this to define an equivalence relation on $\Pi\left(2 n+1, \mathbb{F}_{q}\right)$, then it follows from the combination of [2, Proposition 2.2] and [39, Corollary 4.7] that a supercharacter $\chi_{\Gamma}^{\mathrm{B}}$ for $\Gamma \in \Pi^{\mathrm{B}}\left(n, \mathbb{F}_{q}\right)$ is equal to the restriction of $\chi_{\Lambda}$ if and only if $\Lambda \in \Pi\left(2 n+1, \mathbb{F}_{q}\right)$ belongs to the equivalence class of halve $(\Gamma)$. In turn, it is easy to check that the equivalence class of halve $(\Gamma)$ contains only noncrossing elements if and only if every pair of crossing arcs in $\operatorname{Arc}(\Gamma)$ has the form $(i, j),(-j,-i)$; i.e., if and only if $\Gamma \in \widetilde{\mathrm{NC}^{\mathrm{B}}}\left(n, \mathbb{F}_{q}\right)$. The proof of (c) is similar.

Corollary 7.4. The polynomials $\mathcal{N}_{n}^{\mathrm{B}}(q-1)$ and $\mathcal{N}_{n}^{\mathrm{D}}(q-1)$ count the supercharacters of $\mathbf{U}_{n}^{\mathrm{B}}\left(\mathbb{F}_{q}\right)$ and $\mathbf{U}_{n}^{\mathrm{D}}\left(\mathbb{F}_{q}\right)$ which are not equal to the restriction of any reducible supercharacter of $\mathbf{U}_{2 n+1}\left(\mathbb{F}_{q}\right)$ and $\mathbf{U}_{2 n}\left(\mathbb{F}_{q}\right)$, respectively.

\section{References}

[1] C. A. M. André and A. M. Neto, Supercharacters of finite unipotent groups of types $B_{n}, C_{n}$ and $D_{n}$, J. Algebra 305 (2006), 394-429.

[2] C. A. M. André and A. M. Neto, Supercharacters of the Sylow p-subgroups of the finite symplectic and orthogonal groups, Pacific J. Math. 239 (2009), 201-230. 
[3] C. A. M. André and A. M. Neto, A supercharacter theory for the Sylow $p$-subgroups of the finite symplectic and orthogonal groups, J. Algebra 322, (2009) 1273-1294.

[4] G. E. Andrews, Applications of basic hypergeometric functions, SIAM Rev., 16 (1974) 441-484.

[5] D. Armstrong, Generalized noncrossing partitions and combinatorics of Coxeter groups, Memoirs of the AMS 202 (2009) no. 949.

[6] C. A. Athanasiadis, On noncrossing and nonnesting partitions for classical reflection groups, Electron. J. Combin. 5 (1998), Research Paper 42.

[7] C. A. Athanasiadis and V. Reiner, Noncrossing partitions for the group $D_{n}, S I A M$ J. Discrete Math. 18 (2004), 397-417.

[8] M. Benoumhani, On Whitney numbers of Dowling lattices, Discrete Math. 159 (1996) $13-33$.

[9] M. Benoumhani, On Some Numbers Related to Whitney Numbers of Dowling Lattices, Adv. Appl. Math. 19 (1997) 106-116.

[10] M. Bernstein and N. J. A. Sloane, Some canonical sequences of integers, Linear Algebra Appl. 226/228 (1995), 57-72.

[11] M. Bloss, $G$-colored partition algebras as centralizer algebras of wreath products, $J$. Algebra 265 (2003) 690-710.

[12] J. Bonin, L. Shapiro, and R. Simion, Some $q$-analogues of the Schröder numbers arising from combinatorial statisics on lattice paths, J. Statist. Plann. Inference. 34 (1993) 35-55.

[13] W. Y. C. Chen, E. Y. P. Deng, and R. R. X. Du, Reduction of m-regular noncrossing partitions, European J. Combin. 26 (2005), no. 2, 237-243.

[14] W. Y. C. Chen, E. Deutsch, and S. Elizalde, Old and young leaves on plane trees and 2-Motzkin paths, European J. Combin. 27 (2006) 414-427.

[15] W. Y. C. Chen, A. Y. Z. Wang, and A. F. Y. Zhao, Identities Derived from Noncrossing Partitions of Type B, preprint, arXiv:0908. 2291 (2009).

[16] W. Y. C. Chen, S. H. F. Yan, and L. L. M. Yang, Identities from weighted 2-Motzkin paths, Adv. Appl. Math. 41 (2008), 329-334.

[17] C. Coker, Enumerating a class of lattice paths, Discrete Math. 271 (2003), 13-28.

[18] C. Coker, A family of eigensequences, Discrete Math. 282 (2004), 249-250.

[19] L. Comtet, Advanced Combinatorics, D. Reidel, Dordrecht, 1974.

[20] P. Diaconis and I. M. Isaacs, Supercharacters and superclasses for algebra groups, Trans. Amer. Math. Soc. 360 (2008), 2359-2392.

[21] A. Erdélyi (Ed.), Higher Transcendental Functions, Bateman Project, Vols. 1-3, McGraw-Hill, New York 1953-1955.

[22] E. Goursat, Sur l'équation différentielle linéaire qui admet pour intégrale la série hypergéométrique, Ann. Sci. de l'École Normale Supérieure, X suppl., (1881) 1-142. 
[23] A. Kasraoui, $d$-Regular set partitions and rook placements, Séminaire Lotharingien de Combinatoire 62 (2009) Article B62a, 8pp.

[24] M. Klemm and B. Wagner, A matrix of combinatorial numbers related to the symmetric groups, Discrete Math. 28 (1979), 173-177.

[25] S. Lewis and N. Thiem, Nonzero coefficients in restrictions and tensor products of supercharacters of $U_{n}(q)$, Adv. Math. 227 (2011) 40-72.

[26] T. Mansour and Y. Sun, Identities involving Narayana polynomials and Catalan numbers, Discrete Math. 309 (2009) 4079-4088.

[27] E. Marberg, A supercharacter analogue for normality, J. Algebra 332 (2011), 334365.

[28] R. C. Orellana, On partition algebras for complex reflection groups, J. Algebra 313 (2007) 590-616.

[29] V. Reiner, Non-crossing partitions for classical reflection groups, Discrete Math. 177 (1997) 195-222.

[30] J. Riordan, Combinatorial Identities, John Wiley, New York, 1968.

[31] D. G. Rogers, Rhyming Schemes: Crossings and Coverings, Discrete Math. 33 (1981), $67-77$.

[32] R. Simion, Combinatorial statistics on type-B analogues of noncrossing partitions and restricted permutations, Electron. J. Combin., 7 (2000) R9.

[33] N. J. A. Sloane, editor (2003), The On-Line Encyclopedia of Integer Sequences, published electronically at http://www.research.att.com/njas/sequences/.

[34] M. Z. Spivey, A generalized recurrence for Bell numbers, J. Int. Seq. 11 Article 08.2.5 (2008).

[35] R. P. Stanley, Enumerative Combinatorics, Vol. 2, Cambridge University Press, Cambridge, New York, 1999.

[36] R. A. Sulanke, Bijective recurrences concerning Schröder paths, Electron. J. Combin. 5 (1998), Research Paper 47.

[37] R. A. Sulanke, Bijective recurrences for Motzkin paths, Adv. Appl. Math. 27 (2001) 627-640.

[38] R. A. Sulanke, The Narayana distribution, J. Statist. Plann. Inference 101 (2002) 311-326.

[39] N. Thiem, Branching rules in the ring of superclass functions of unipotent uppertriangular matrices, J. Algebr. Comb. 31 (2009), 267-298. 